\title{
Automated Analysis of Nerve-Cell Images Using Active Contour Models ${ }^{1}$
}

\author{
Ying-Lun Fok ${ }^{\S}$, Joseph Chan $\stackrel{\ddagger}{\ddagger}$ and Roland T. Chin $§ \dagger$ \\ $\dagger$ Electrical \& Computer Engineering \\ University of Wisconsin, Madison, Wisconsin 53706 \\ $\S$ Computer Science Department \\ Hong Kong University of Science \& Technology \\ $\ddagger$ Division of Technology \\ City University of Hong Kong
}

Keywords: Active Contour Model, Snakes, Snake Initialization, Cell Analysis, Axon

\begin{abstract}
The number of nerve fibers (axons) in a nerve, the axon size and shape can all be important neuroanatomical features in understanding different aspects of nerves in the brain. However, the number of axons in a nerve is typically in the order of tens of thousands and a study of a particular aspect of the nerve often involves many nerves. Potentially meaningful studies are often prohibited by the huge number involved when manual measurements have to be employed. A method that automates the analysis of axons from electronmicrographic images is presented. It begins with a rough identification of all the axon centers by use of an elliptical Hough transform procedure. Boundaries of each axons are then extracted based on active contour model, or snakes, approach where physical properties of the axons and the given image data are used in an optimization scheme to guide the snakes to converge to axon boundaries for accurate sheath measurement. However, false axon detection is still common due to poor image quality and the presence of other irrelevant cell features, thus a conflict resolution scheme is developed to eliminate false axons to further improve the performance of detection. The developed method has been tested on a number of nerve images and its results are presented.
\end{abstract}

\footnotetext{
${ }^{1}$ Supported by a grant from Sino Software Research Center.
} 


\section{INTRODUCTION}

The brain of human or other mammals consists of billions of nerve cells called neurons. Each neuron, in turn, connects with distant neurons through projection nerve fibers called axons. Information in the form of electrical pulses are transmitted between neurons through their connected axons where they are bundled together forming a nerve.

With the huge number of neurons in the brain (a rough estimate of ten billions), any reliable quantitative estimate of the number of neurons or axons even in a small functional unit or from an anatomical nucleus of the brain is a very formidable, and often impractical, task if done manually. Yet, a quantitative analysis of the number of neurons is very useful in understanding the normal, diseased, developing, aging, or injured brain [3].

Since a nucleus is a three-dimensional cellular organelle, counting neurons in a nucleus involves sectioning the nucleus into many sections. The thickness of which is typically less than the diameter of each neuron. The number of neurons in each two-dimensional section is counted and the total obtained by summing across sections. However, one has to account for part of each neuron appearing in more than one section. It is therefore easier to work with the axons of those neurons since a single two-dimensional section of the axon bundle will indicate the neuron count.

Besides the axon count, there are many other morphological factors that do play a role in brain functions. For example, if the sheath wrapping around the axons (myelin sheath) deteriorates, the brain will suffer from multiple sclerosis. Also, the diameter of the axon is known to affect conduction rate of the electrical pulses transmitted by the axon. All these quantities are important to the study of brain functions and diseases.

Traditionally, the axon counting process was done manually by experienced neuroscientists. However, since each nerve bundle contains a huge number of axons, this approach is extremely time consuming and ineffective. As a result, there is a strong motivation to

have such counting process automated. The application of computer image processing to cell counting/recognition has drawn much attention from image processing and cell biology communities $[20,17,13,7,18,10,6]$.

The majority of the cell counting algorithms proposed so far are based on simple thresholding techniques $[7,11,10,6,18]$. It assumes that the target cellular objects exhibit certain intensity gray levels which are distinguishable from the background and a simple thresholding is adequate to extract the desired components. For instance, the intensity of cell nuclei, which is darker than the rest of the cell bodies, can serve as a clue for extraction [6, 7]. In case of color images, each color component may be used to extract different types of cells [18]. The thresholds required by these algorithms may be either assigned by experts or computed automatically from a set of training data. In the latter case, thresholds are usually estimated by histogram analysis [11] or by maximizing certain interclass variance functions [10]. After 
thresholding, grouping processes then define different regions as the maximal connected sets of pixels, each with the same label. However, thresholding techniques are, in general, very sensitive to imaging variations, such as lighting and sectioning thickness of the nerve, making them unreliable.

There exists a few sophisticated cell analysis algorithms. In one system multiple thresholding and efficient search are used [6], however, it only works for isolated, non-touching cells. In another system [13], shape information of the cells is used to split cell clumps into individual cells where clumps are first segmented by edge detection, skeletonization, region growing and labeling. This approach works well if there is little variation in cell shapes.

The problem we study here involves automated analysis of electronmicrograph axon images, where axon counts, their size, diameters, and myelin thickness distributions are the desired outputs. Fig. 5(a) shows a typical axon image. Each cellular object surrounded by a dark wrapping (myelin sheath) indicates an axon. The difficulties of traditional cell counters for this type of images include:

1. The lack of prominent features, such as a single nucleus or high contrast, provides no indication of the presence of an axon.

2. The presence of closely-packed, touching axons renders the usefulness of traditional contour tracing or edge detection.

3. The highly irregular axon shapes makes traditional template matching or Hough transform infeasible,

The aforementioned difficulties make extraction, recognition of axons and subsequently shape analysis difficult. Therefore, axon detection can only be done by tracing the myelin sheaths. In this paper, the active contour model, commonly known as snakes, first introduced in 1987 [14] is used to detect and measure axons. Snakes have the advantage of combining data-driven and model-driven approaches in a unified manner, overcoming the difficulties of lack of prominent features and irregularities in shape.

The snake approach has been adapted to suit our particular application, which includes:

1. A boundary estimate, or initial guess, is placed near each axon boundary for the snake to fine-tune to its final accurate solution. The initialization process requires no user interaction and is able to handle shape irregularities and image noise.

2. Prior knowledge of the axon is incorporated into the snake's energy functionals for the detection of the optimal boundary.

3. To avoid both under-counting and false detection, a post-processing stage to resolve conflicts and eliminate false detections is developed so that the final result is insensitive to the initial guess or other parameters. 
The remaining of this paper is organized as follows. Section 2 reviews the snake formulation. Section 3 gives an overview of the overall system. Section 4 describes image preprocessing. In Section 5, we introduce a transform approach to snake initialization. The energy measures for the snake are formulated in Section 6. The post-processing for false alarm elimination, based on the notion of conflict, is described in Section 7, and then follows by experimental results. Section 9 gives the conclusion.

\section{ACTIVE CONTOUR MODEL}

An active contour can be considered as an energy-minimizing spline which searches for local energy minimum on a potential surface specified by a number of application-specific energy functionals representing prior knowledge of the object boundaries being sought [14, 16]. After placing an initial snake on the image, it moves automatically to the physical object boundary under the influence of the energy functionals. The name snake comes from the way that it slithers while minimizing its energy.

A snake $\mathcal{V}$ is a set of points, or snaxels, $\left\{\mathbf{v}_{i} \mid i=1,2, \cdots, n\right\}$ representing a boundary. The total energy of a snake is contributed from a number of energy functionals, $E_{1}, E_{2}, \cdots, E_{k}$, each reflects a particular characteristic of the boundary. The significance of each functional $E_{r}$ is weighted by the corresponding regularization parameter ${ }^{2} \lambda_{r}$. The total energy of a snake $\mathcal{V}$ is the weighted sum of these energy terms, i.e.,

$$
E_{\text {snake }}(\mathcal{V})=\sum_{r=1}^{k} \lambda_{r} E_{r}(\mathcal{V})
$$

where $\sum_{r=1}^{k} \lambda_{r}=1$ (see footnote $\left.{ }^{3}\right)$.

Each energy functional is, in turn, equal to the aggregation of snaxel energy over the entire snake, i.e.,

$$
E_{r}(\mathcal{V})=\sum_{i=1}^{n} e_{r}\left(\mathbf{v}_{i}\right)
$$

where $e_{r}(\cdot)$ is the snaxel energy with respect to the energy functional $E_{r}$. The dynamics of snakes is completely governed by the set of functionals $\left\{E_{r}\right\}_{r=1,2, \cdots, k}$, as well as their respective regularization parameters $\lambda_{r}$ 's.

\footnotetext{
${ }^{2}$ The snake formulation is viewed as an ill-posed inverse problem in computer vision (see [23]) in which conflicting constraints are used to reach for a compromised solution. The standard term regularization "parameters" is used in this paper. Other terms, such as weights and smoothing parameters, are also commonly used by others.

${ }^{3}$ Since the convergence of snakes only depends on relative values of the weights, summing up all the weights to one is mainly for clarity in presentation.
} 
Having defined the snake model, boundary detection becomes finding the snake $\mathcal{V}^{*}$ such that $E_{\text {snake }}\left(\mathcal{V}^{*}\right)$ is minimized, i.e.,

$$
\mathcal{V}^{*}=\arg \min _{\mathcal{V}} E_{\text {snake }}(\mathcal{V})
$$

In this respect, the active contour model transforms boundary detection into an optimization problem. This energy minimization has been solved by calculus of variations [14], dynamic programming [1] and greedy algorithm [24].

The energy functionals can basically be grouped into two classes: $E_{\text {int }}$ and $E_{\text {ext }}$. The internal energy, $E_{\text {int }}$, depends solely on the prior knowledge of the boundary model, regardless of the actual image data. For examples, continuity of the boundary to ensure a smooth spline may be encoded in $E_{\text {int }}$. On the other hand, the external energy $E_{\text {ext }}$ represents the data dependent component of the energy term, which involves image data, usually in form of image intensity gradients. The snake energy can be concisely written in terms of these two energy types as

$$
E_{\text {snake }}(\mathcal{V})=\lambda E_{\text {int }}(\mathcal{V})+(1-\lambda) E_{\text {ext }}(\mathcal{V}) \text { where } \lambda \in[0,1]
$$

Minimizing $E_{\text {int }}(\mathcal{V})$ favors the snake to conform to a prior model while a low $E_{\text {ext }}(\mathcal{V})$ means that the detected boundary is conformed to salient image features. In this respect, $E_{i n t}$ and $E_{e x t}$ represent the model-driven and the data-driven components of the snake, respectively. The significance of each component is determined by the regularization parameter $\lambda$. When $\lambda \approx 0, E_{\text {ext }}(\mathcal{V})$ dominates the energy expression in Eq. (4), thus reducing the snake to a data-driven technique such as contour tracing. In the other extreme, when $\lambda \approx 1$, the snake converges to the shape that minimizes $E_{\text {int }}(\mathcal{V})$ where the solution is a form of template matching. See [15] for some examples.

In between these extremes, the snake balances between template matching and contour tracing. In the case where no prior knowledge is available for selecting a suitable $\lambda$, a local minimax criterion as proposed in [15] may be used, whereby values of $\lambda$ can be automatically and implicitly determined without depending on any input parameter or heuristic.

\section{ALGORITHM OVERVIEW}

The cell counting and sizing procedure is summarized by the following steps which are described in the subsequent sections:

1. Morphological filtering to remove axoplasmic organelles inside axons.

2. Elliptical Hough transform to locate interior points.

3. Inner snake initialization using each interior point.

4. Energy minimization for inner snakes. 
5. Outer snake initialization using each inner snake.

6. Energy minimization for outer snakes.

7. Conflict resolution to detect true axons and eliminate false alarms.

8. Physical measurements

\section{PREPROCESSING}

The input axon image is a 1:9000 gray scale electronmicrographic image. See Fig. 2(a) and 5(a) for examples. A major problem in axon detection is the presence of axoplasmic organelles like mitochondria which would lead to errors in snake initialization and false detection. It is highly desirable to remove these irrelevant features before detection and analysis, and that is achieved by morphological filtering presented in the following.

Let $I(\mathbf{r})$ denotes the image intensity at location $\mathbf{r}$. A direct method to remove the axoplasmic organelle is to close $I(\mathbf{r})$ with a flat structuring element larger than the axoplasmic organelle. However, thin myelin sheaths with comparable thickness to that of axoplasmic organelles may also be removed by the filter.

A multidirectional filter [22] is used to overcome this problem. Let $L^{\ell}(\mathbf{r})$ denote a linear structuring element with length $\ell$ which is chosen to be greater than the typical dimension of axoplasmic organelles. Let $D$ be its region of support and define $L^{\ell}(\mathbf{r})=0$ for all $\mathbf{r} \in D$.

Since $L^{\ell}$ is longer than the axoplasmic organelles, they are removed by a closing operation •[·.]. On the other hand, $L^{\ell}$ is 1-pixel thick and has no effect on the portion of myelin sheaths that run parallel to $L^{\ell}$. Consequently, only axoplasmic organelles are removed and properlyaligned thin sheath structures are preserved.

To preserve thin sheaths in all directions, the above operation is repeated at multiple directions by applying directional elements, each to the original image. We define $\eta$ similar structuring elements, $L_{i}^{\ell}(\mathbf{r})$, where $i=1,2, \ldots, \eta$ denotes the particular direction. The axoplasmic organelle removal operation becomes:

$$
\begin{aligned}
\xi_{i}^{\ell}(\mathbf{r}) & =I(\mathbf{r}) \bullet L_{i}^{\ell}(\mathbf{r}) \\
& =\min _{\boldsymbol{\alpha} \in D_{i}} \max _{\boldsymbol{\beta} \in D_{i}} I(\mathbf{r}+\boldsymbol{\alpha}-\boldsymbol{\beta}) \\
g(\mathbf{r}) & =\min _{i \in[1, \eta]}\left\{\xi_{i}^{\ell}(\mathbf{r})\right\}
\end{aligned}
$$

where $D_{i}$ is the region of support of $L_{i}^{\ell}(\mathbf{r})$ and $g(\mathbf{r})$ selects the output amongst the multiple directions.

Suppose that the sheath is parallel to $L_{i}^{\ell}$ at $\mathbf{r}_{0}, \xi_{i}^{\ell}\left(\mathbf{r}_{0}\right)$ will yield the lowest intensity, roughly the same as the original intensity at that point, among all other $\xi_{j}^{\ell}\left(\mathbf{r}_{0}\right)$ 's, where $i \neq j$, thus $\xi_{i}^{\ell}$ will dominate the output $g\left(\mathbf{r}_{0}\right)$ according to Eq. (6). On the other hand, if 
the axoplasmic organelle dimension is smaller than $\ell$, it is removed completely, and narrow structures parallel to $L_{i}^{\ell}$ are preserved.

A similar multidirectional filter, with closing replaced by opening and minimum replaced by maximum, is employed to fill small holes found inside the myelin sheath.

In our implementation, four elements at $0^{\circ}, 45^{\circ}, 90^{\circ}$ and $135^{\circ}$, respectively are used, and $\ell=16$ pixels for axoplasmic organelle removal and $\ell=7$ pixels for hole filling are chosen. See Fig. 2(b) and 5(b) for examples of preprocessing.

\section{INITIALIZATION OF SNAKES}

The active contour model approach is an iterative energy minimization process which starts from an initial guess. If an initial boundary is placed too far from the solution boundary, the snake might converge erroneously to an undesirable shape. The initialization issue is application specific, requiring either prior knowledge or user interaction $[15,4,26]$. In this section, we propose an automated initialization process for the nerve-cell images.

Each axon comprises of an inner boundary and an outer boundary. The objective of initialization is to determine two estimates, namely $\tilde{\mathcal{V}}_{\text {in }}$ and $\tilde{\mathcal{V}}_{\text {out }}$, which are sufficiently close to the true inner and outer boundaries, respectively.

\subsection{Hough Transform to Locate Potential Axons}

The axon boundary is highly irregular and varies considerably from one to another. Any sort of geometric functions are inadequate to characterize them, or to place an initial snake close to the true solution. With the same reasons, a generalized Hough transform [2], which requires a set of representative shapes to train for the final recognition is also infeasible. In view of this, we propose to first locate all the potential axons and then to construct an initial boundary snake based on each potential candidate.

The inner boundary of an axon defines a closed set in which every element of that set represents a location inside the axon. Let us call such an element an interior point ${ }^{4}$ which can be used as a cue to indicate the presence of an axon. Initialization of an inner snake is followed after the detection of such an interior point.

It is observed that an elliptical shape provides a very crude representation of axons, thus can be used to detect axon interior points and in turn to detect potential axons. It is to be noted that the detection of potential axons only serves as the beginning of the automated process which subsequently refines the detected candidates for accurate detection and measurement. With the elliptical representation, we formulate the following objective: Given an elliptical boundary $B$, we aim to locate a point $\mathbf{p}$ interior to $B$.

\footnotetext{
${ }^{4} \mathrm{~A}$ point $\mathbf{p}$ is an interior point of a set $B$ if there is a neighborhood $N$ of $\mathbf{p}$ such that $N \subset B$.
} 


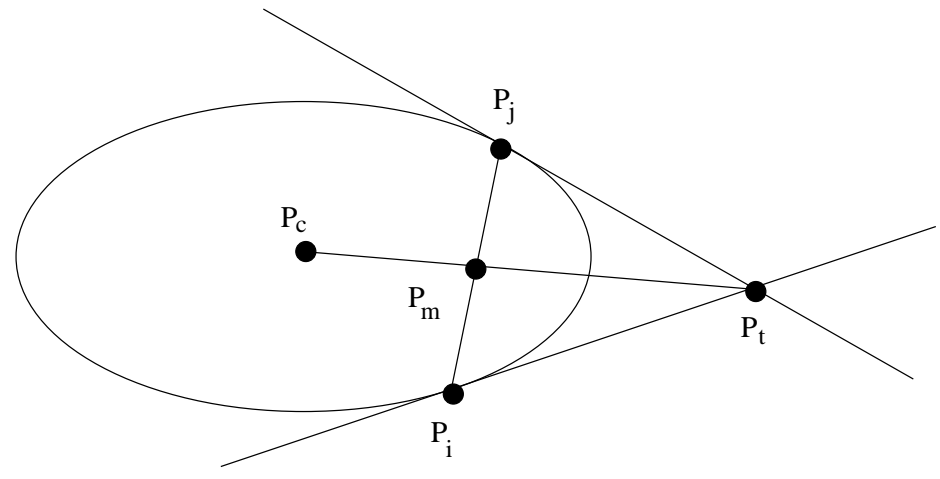

Figure 1: Ellipse showing that $P_{c}, P_{m}$ and $P_{t}$ are collinear.

A Hough transform for elliptical shape [8] is applied to accumulate local evidence derived from discrete boundary edges in order to infer the location of an interior point. Let $\mathbf{p}_{i}$ and $\mathbf{p}_{j}$ be any two points on an ellipse with $\mathbf{p}_{c}$ as center and $\mathbf{p}_{m}$ as the mid-point between $\mathbf{p}_{i}$ and $\mathbf{p}_{j}$ (Fig. 1). The equations of tangents at $\mathbf{p}_{i}$ and $\mathbf{p}_{j}$ respectively can be written as:

$$
\begin{aligned}
\mathcal{L}_{i}: \mathbf{r}(\alpha) & =\mathbf{p}_{i}+\alpha \mathbf{t}_{i} \\
\mathcal{L}_{j}: \mathbf{r}(\beta) & =\mathbf{p}_{j}+\beta \mathbf{t}_{j}
\end{aligned}
$$

where $\mathbf{t}_{i}$ and $\mathbf{t}_{j}$ are the tangent vectors at $\mathbf{p}_{i}$ and $\mathbf{p}_{j}$, and they can be estimated from the image intensity gradient direction at their respective locations. When $\mathbf{t}_{i} \neq \mathbf{t}_{j}$, there exist $\alpha^{\prime}$ and $\beta^{\prime}$ such that $\mathbf{p}_{i}+\alpha^{\prime} \mathbf{t}_{i}=\mathbf{p}_{j}+\beta^{\prime} \mathbf{t}_{j}=\mathbf{p}_{t}$, the intersection between $\mathcal{L}_{i}$ and $\mathcal{L}_{j}$. As $\alpha^{\prime}$ and $\beta^{\prime}$ can be solved explicitly from Eq. (7) and Eq. (8), $\mathbf{p}_{t}$ can be determined. Hence the straight line passing through $\mathbf{p}_{t}$ and $\mathbf{p}_{m}$ can be parameterized in terms of $\mu$ as:

$$
\mathbf{r}_{i j}(\mu)=\mathbf{p}_{m}+\mu\left(\frac{\mathbf{p}_{m}-\mathbf{p}_{t}}{\left\|\mathbf{p}_{m}-\mathbf{p}_{t}\right\|}\right)
$$

The geometric property of ellipse guarantees that the center $\mathbf{p}_{c}$ always lies in the locus of $\mathbf{r}_{i j}(\mu)$ [25]. In other words, there exists an $\mu_{c}^{(i j)}$ such that

$$
\begin{aligned}
\mathbf{r}_{i j}\left(\mu_{c}^{(i j)}\right) & =\mathbf{p}_{m}+\mu_{c}^{(i j)}\left(\frac{\mathbf{p}_{m}-\mathbf{p}_{t}}{\left\|\mathbf{p}_{m}-\mathbf{p}_{t}\right\|}\right) \\
& =\mathbf{p}_{c}
\end{aligned}
$$

For every two points $\mathbf{p}_{i}$ and $\mathbf{p}_{j}$ lying on an ellipse, we can thus specify a set of points, denoted by $P_{i j}$, such that

$$
P_{i j}=\left\{\mathbf{r}_{i j}(\mu) \mid \mu \in\left[0, \mu_{\max }\right]\right\}
$$

where $\mu_{\max }>\mu_{c}^{(i j)}$ for all $i, j$. In practice, we set $\mu_{\text {max }}$ to be greater than the maximum length of the semi-major axis among all potential ellipses, so that their centers are guaranteed to fall in the set $P_{i j}$. 
In our implementation, local evidence for an interior point is gathered in a two-dimensional array called the H-space. Suppose we quantize the set $P_{i j}$, and then increment those intersecting H-space arrays by the amount equal to $\left|\overline{\nabla I\left(\mathbf{p}_{i}\right)}\right|\left|\overline{\nabla I\left(\mathbf{p}_{j}\right)}\right|$, the product of normalized intensity gradient magnitudes ${ }^{5}$ of point $\mathbf{p}_{i}$ and $\mathbf{p}_{j}$. If the same procedure is repeated for every two points lying on an ellipse, the H-space array will manifest a peak at the location containing $\mathbf{p}_{c}$ since $\mathbf{p}_{c}$ must be included in every set $P_{i j}$, no matter which two points are chosen. More specifically, if there are $N$ points on an ellipse and each has edge magnitude equal to 1 , the height of the H-space peak will be $\frac{N(N-1)}{2}$.

The contribution of each pair of points to the H-space is proportional to the product of their gradients, therefore practically only those points which are near or at an edge can contribute to the H-space. With an understanding of this, we can neglect those points which have small gradient in generating the H-space. This gradient threshold only affects the speed of the algorithm but not the resulting $\mathrm{H}$-space. Moreover, since the rough size of the axon is known, pixel pairs that are far apart with respect to the known axon dimension are also neglected in the construction of the H-space.

This procedure can be interpreted as a transformation from an image space to a parameter space. Each pair of local features (boundary pixels) votes for all possible ellipse centers and the votes cast by every pair are accumulated in an accumulator array. Since the center of an ellipse is a legitimate interior point, interior point localization is reduced to peak searching in the H-space.

Although an ellipse is far from an adequate axon model, each segment of an axon boundary does represent a piece of an ellipse which contribute to a peak in the H-space. After all the axon boundaries in the image voted in the $\mathrm{H}$-space according to the aforementioned elliptical model, the peaks in the H-space are then considered to be the potential axon interior points. The selection of peaks in the $\mathrm{H}$-space is done in the order of their respective strength (votes), where each peak corresponds to a new snake to be initialized. In the actual implementation, a minimum peak strength has to be imposed to specify the stopping condition for peak searching. This minimum peak strength, in turn, would affect the number of snakes to be initialized. But as described in a later section, the detected axons will be further processed by a conflict resolution procedure to eliminate false detections, thus the number of peaks to be processed only affects the speed of the algorithm, but is insensitive to the final outcome.

Figures 2(c) and 5(c) show some examples. The interior point localization procedure is fairly tolerant to image noise and axon shape variation.

\footnotetext{
${ }^{5}|\overline{\nabla 1}|$ is the intensity gradient normalized to $[0,1]$ by dividing the $98 \%$ quartile intensity gradients and truncating the remaining $2 \%$ to 1 . This, in effect, eliminates most outliers.
} 


\subsection{Inner Snake Initialization}

Once an interior point of an axon has been located, an inner boundary snake is initialized by projecting the interior point outward towards the inner boundary $[5,19]$ at all directions, each with a certain angular increment. At each direction, the projection stops at the nearest location $\mathbf{r}$ where a significant image edge is encountered, that is, $|\overline{\nabla I(\mathbf{r})}|>T_{\text {edge }}$, where $T_{\text {edge }}$ is the expected minimum edge strength of the inner axon boundary. Then a snaxel is initialized at $\tilde{\mathbf{u}}_{i}=\mathbf{r}$. Combining all initialized snaxels, this strategy yields an initial inner snake $\tilde{\mathcal{V}}_{i n}=\left\{\tilde{\mathbf{u}}_{i} \mid i=1,2, \ldots, n\right\}$ that is expected to be in the vicinity of the true boundary.

Since the intensity gradient along the inner boundaries is substantially greater than that of the background, $T_{\text {edge }}$ can be taken from a wide range without noticeable effect on $\tilde{\mathcal{V}}_{i n}$ and this has been verified experimentally. For cases where there are much noise and other clutters inside an axon, or a badly chosen $T_{e d g e}$, some initialized snaxels will be misplaced. However, the initialization is to obtain a rough estimate, rather than an accurate boundary, for further extraction of the axon boundary. The misplaced snaxels will be corrected after the snake converges to the true boundary; the process is described in Section 6 .

Figure 2(d) shows an example of the initial snake. Note that three snaxels are misplaced near the axoplasmic organelle. Nevertheless, the converged snake in Fig. 2(e) is able to detect the correct inner boundary.

\subsection{Outer Snake Initialization}

The initial outer snake $\tilde{\mathcal{V}}_{\text {out }}$ is estimated after the determination of the converged inner snake $\mathcal{V}_{i n}$. It is obtained by expanding $\mathcal{V}_{i n}$ a distance $t^{*}$ uniformly outward due to the fact that the inner and outer boundaries of each axon bear similar shape. Specifically, $\tilde{\mathcal{V}}_{\text {out }}$ is obtained by

$$
\tilde{\mathcal{V}}_{\text {out }}=\left\{\tilde{\mathbf{v}}_{i}=\mathbf{u}_{i}+t^{*} \hat{\mathbf{d}}_{i} \mid \mathbf{u}_{i} \in \mathcal{V}_{\text {in }}, i=1,2, \cdots, n\right\}
$$

where $\hat{\mathbf{d}}_{i}$ represents the perpendicular outward vector measured at $\mathbf{u}_{i}$ given by

$$
\begin{aligned}
\hat{\mathbf{d}}_{i} \cdot\left(\mathbf{u}_{i+1}-\mathbf{u}_{i-1}\right) & =0 \\
& =1 \\
\hat{\mathbf{d}}_{i} \cdot \frac{\left\|\hat{\mathbf{d}}_{i}\right\|}{\nabla I\left(\mathbf{u}_{i}\right)} & <0
\end{aligned}
$$

The distance $t^{*}$ is estimated based on the observation that myelin thickness of each individual fiber is roughly uniform. It is determined by constructing a histogram $T$ representing the distribution of outer edge distance from the inner snake $\mathcal{V}_{i n}$ to the outer boundary edges. For each inner snaxel $\mathbf{u}_{i}$, the edge strength along the direction specified by $\hat{\mathbf{d}}_{i}$ is examined. For every point $\mathbf{r}$ on the line segment

$$
\mathbf{r}=\mathbf{u}_{i}+t \hat{\mathbf{d}}_{i}
$$


where $t \in\left[t_{\min }, t_{\max }\right]$, the histogram $T$ is incremented at $t$ by $|\overline{\nabla I(\mathbf{r}(t))}|$. The endpoints $t_{\min }$ and $t_{\text {max }}$ are taken to be the expected minimum and maximum thickness of myelin sheath. Repeating for every inner snaxel, the distribution of edge distance away from $\mathcal{V}_{\text {in }}$ is estimated. The histogram $T$ exhibits a peak at $t^{*}$ which indicates the most likely myelin thickness. After the determination of $t^{*}$, the initial outer snake $\tilde{\mathcal{V}}_{\text {out }}$ is completely specified by Eq. (12). Figures 2(f) and 5(f) show some examples.

\section{FORMULATION OF ENERGY FUNCTIONALS}

The initial inner and outer snakes, $\tilde{\mathcal{V}}_{\text {in }}$ and $\tilde{\mathcal{V}}_{\text {out }}$, respectively are only gross estimates. They are further refined by the active contour model approach. An active contour model is formulated in the context of energy minimization. The following specifies the energy functionals used in this application, with the first three adopted from those of [15].

\subsection{Continuity Constraint}

The snaxel continuity energy [15], $e_{\text {cont }}$, at point $\mathbf{v}_{i}$ is defined as:

$$
e_{\text {cont }}\left(\mathbf{v}_{i}\right)=\frac{\left(\bar{d}-\left\|\mathbf{v}_{i}-\mathbf{v}_{i-1}\right\|_{\infty}\right)^{2}}{\bar{d}^{2}}
$$

where $\|\mathbf{v}\|_{\infty}$ denotes the infinity norm, i.e., $\max \left\{\left|v_{x}\right|,\left|v_{y}\right|\right\}$, and $\bar{d}$, which is updated at every iteration, is an average inter-snaxel distance measure.

The continuity functional favors a snake which has evenly-spaced snaxels. If every pair of adjacent snaxels has a separation of $\bar{d}, E_{\text {cont }}(\mathcal{V})=0$. Conversely, $E_{\text {cont }}(\mathcal{V})$ increases as separation varies highly. Since the deviation measure is normalized by $\bar{d}$, $e_{\text {cont }}$ is invariant to scaling, thus preventing contours from either expanding or shrinking.

\subsection{Curvature Constraint}

The curvature constraint [15] prohibits a snake from acute bending and the curvature energy associated with each snaxel is defined as:

$$
e_{\text {curve }}\left(\mathbf{v}_{i}\right)=\frac{1}{4}\left|\frac{\Delta \mathbf{v}_{i}}{\left\|\Delta \mathbf{v}_{i}\right\|}-\frac{\Delta \mathbf{v}_{i-1}}{\left\|\Delta \mathbf{v}_{i-1}\right\|}\right|
$$

where $\Delta \mathbf{v}_{i}=\mathbf{v}_{i+1}-\mathbf{v}_{i}$. It can be shown that

$$
e_{\text {curve }}\left(\mathbf{v}_{i}\right)=\frac{1}{2}\left(1-\cos \left(\psi_{i}\right)\right)
$$

where $\psi_{i}$ is the bending angle at $\mathbf{v}_{i}$ and $e_{\text {curve }}$ is normalized to $[0,1]$. When $\mathbf{v}_{i-1}, \mathbf{v}_{i}$, and $\mathbf{v}_{i+1}$ lie in a straight line, $\psi_{i}=0$, and thus $e_{\text {curve }}\left(\mathbf{v}_{i}\right)=0$. On the other hand, if these three points form a sharp angle, $\psi_{i} \approx \pi$ and $e_{\text {curve }}\left(\mathbf{v}_{i}\right) \approx 1$. Therefore, $E_{\text {curve }}(\mathcal{V})$ can be 


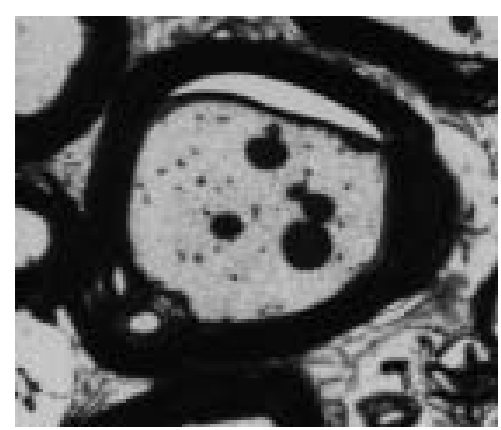

$2(\mathrm{a})$

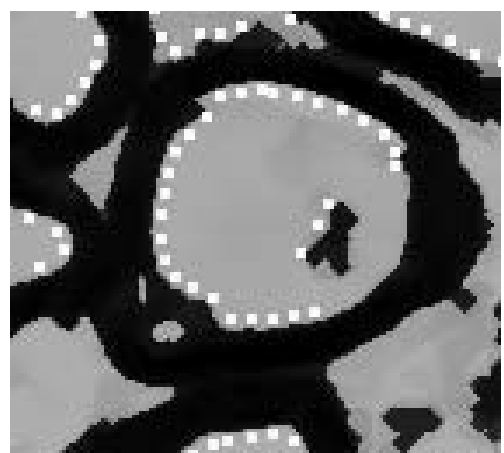

$2(d)$

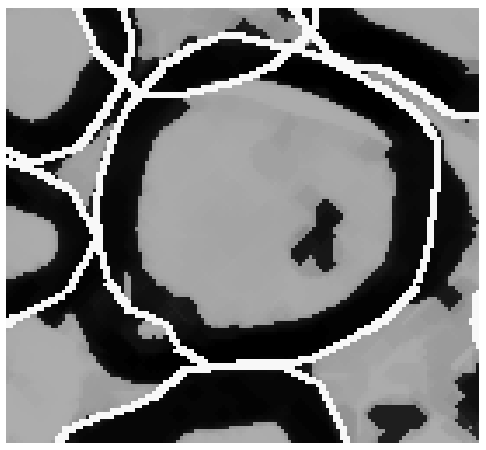

$2(g)$

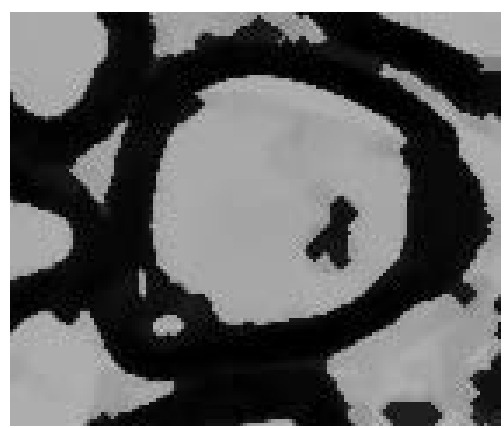

2(b)

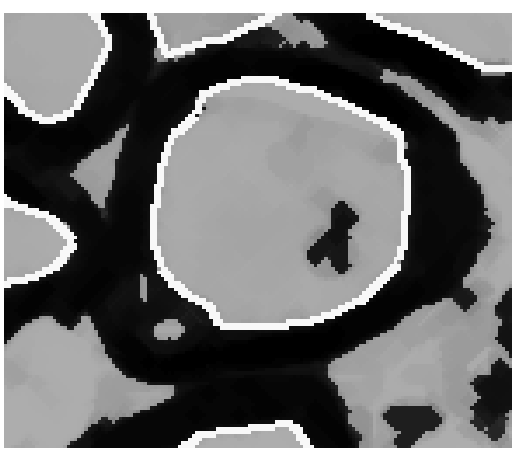

$2(\mathrm{e})$

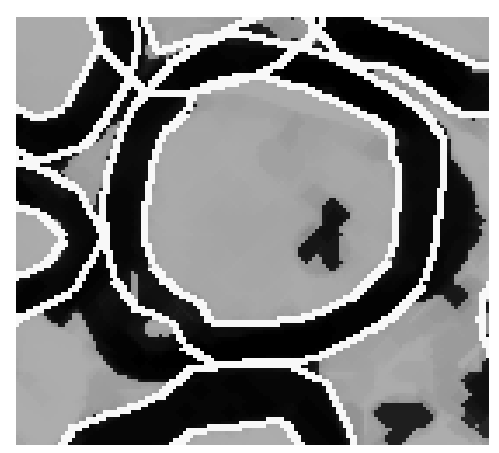

$2(h)$

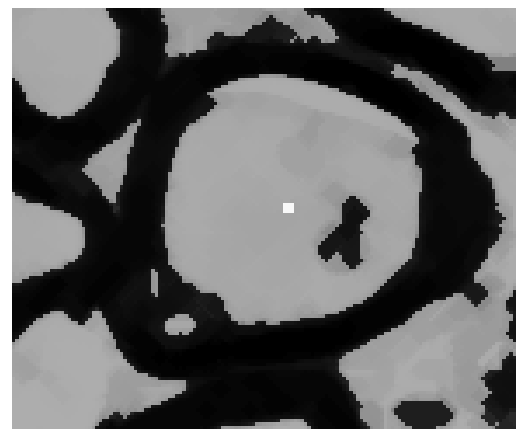

2 (b)

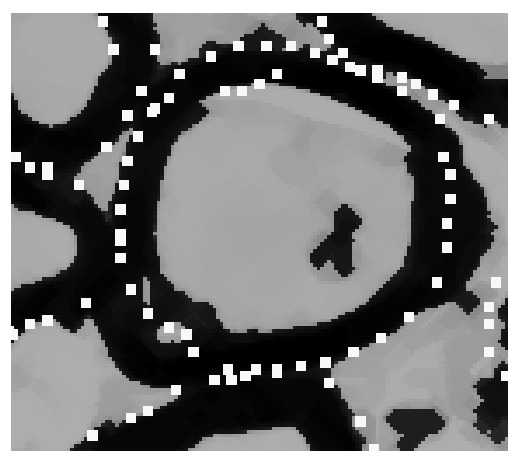

$2(f)$

Figure 2: Sequence of a single-cell image: (a) Original. (b) Preprocessed image $g(\mathbf{r})$. (c) Detected interior point. (d) Initial inner snake $\tilde{\mathcal{V}}_{i n}$. (e) Converged inner snake $\mathcal{V}_{i n}$. (f) Initial outer snake $\tilde{\mathcal{V}}_{\text {out }}$. (g) Converged outer snake $\mathcal{V}_{\text {out }}$. (h) All converged snakes; the outer snake of the center axon failed to capture part of the outer boundary due to the application of the equal thickness constraint. This affects the accuracy of the myelin thickness distribution measure. 
considered as a measure of smoothness of a snake. This constraint is particularly relevant to axons due to the smooth nature of cell boundaries.

\subsection{Edge Constraint}

The functional responsible for attracting a snake to the image boundary is expressed as [15]:

$$
e_{e d g e}\left(\mathbf{v}_{i}\right)=1-\left|\overline{\nabla I\left(\mathbf{v}_{i}\right)}\right|\left(1-\left|\cos \left(\varphi_{i}\right)\right|\right)
$$

where $\varphi_{i}$ is the angle between the intensity gradient vector and the unit tangent vector at $\mathbf{v}_{i}$ (Fig. 3).

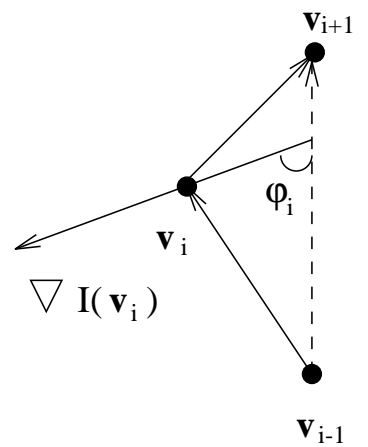

Figure 3: Notations used in the formulation of $e_{e d g e}$

Obviously, when $\mathbf{v}_{i}$ is located on an edge, $e_{e d g e}$ is minimized. The incorporation of gradient direction $\varphi_{i}$ into $e_{e d g e}$ has the effect of enforcing consistency of edge orientation of a snake to the underlying image intensity. The factor $1-\left|\cos \left(\varphi_{i}\right)\right|$ weights the magnitude of the intensity gradient continuously against any orientation inconsistency. In particular, $e_{e d g e}\left(\mathbf{v}_{i}\right)$ attains the minimum value of 0 if $\left|\overline{\nabla I\left(\mathbf{v}_{i}\right)}\right|=1$ and $\varphi_{i}=\pi / 2$, while it will reach the maximum value of 1 when either $\left|\overline{\nabla I\left(\mathbf{v}_{i}\right)}\right|=0$ or $\varphi_{i}=0$.

\subsection{Equal Thickness Constraint}

Many fibers are closely clustered together to such an extent that their boundaries are not resolvable. The above three functionals alone [15] will not be able to extract these adjoined boundaries. To resolve them, a specific functional is proposed to encourage equal thickness between the inner and outer snakes.

As described in Section 5.3, for each inner snaxel $\mathbf{u}_{i}$, an outer snaxel $\mathbf{v}_{i}$ is initialized at $\mathbf{u}_{i}+t^{*} \hat{\mathbf{d}}_{i}$. In addition, a force of attraction between $\mathbf{u}_{i}$ and $\mathbf{v}_{i}$, denoted by $e_{\text {thick }}\left(\mathbf{v}_{i}\right)$, is used to relate the inner and outer boundaries. Let $\bar{t}$ be the average thickness of the axon under consideration, $\hat{\mathbf{d}}_{i}$ be the unit outward vector as in Eqs. (13) - (15). The average thickness 
$\bar{t}$ is updated iteratively as the snake converges. The relation between $\mathcal{V}_{i n}$ and $\mathcal{V}_{\text {out }}$ specified by $e_{\text {thick }}$ has the following properties:

1. Location: $e_{\text {thick }}\left(\mathbf{v}_{i}^{*}\right)=0$ when $\mathbf{v}_{i}^{*}=\mathbf{u}_{i}+\bar{t} \hat{\mathbf{d}}_{i}$

2. Direction: $e_{t h i c k}\left(\mathbf{u}_{i}+t \hat{\mathbf{d}}^{\prime}\right)>e_{\text {thick }}\left(\mathbf{u}_{i}+t \hat{\mathbf{d}}^{\prime \prime}\right)$ if and only if $\left|\phi^{\prime}\right|>\left|\phi^{\prime \prime}\right|$, where $\hat{\mathbf{d}}^{\prime}$ and $\hat{\mathbf{d}}^{\prime \prime}$ are unit vectors that deviate from $\hat{\mathbf{d}}_{i}$ by an angle of $\phi^{\prime}$ and $\phi^{\prime \prime}$, respectively.

3. Displacement: $e_{\text {thick }}\left(\mathbf{u}_{i}+\left(\bar{t}+\delta^{\prime}\right) \hat{\mathbf{d}}^{\prime}\right)>e_{\text {thick }}\left(\mathbf{u}_{i}+\left(\bar{t}+\delta^{\prime \prime}\right) \hat{\mathbf{d}}^{\prime}\right)$ if and only if $\left|\delta^{\prime}\right|>\left|\delta^{\prime \prime}\right|$ and

4. Displacement Symmetry: $e_{\text {thick }}\left(\mathbf{u}_{i}+(\bar{t}+\delta) \hat{\mathbf{d}}^{\prime}\right)=e_{\text {thick }}\left(\mathbf{u}_{i}+(\bar{t}-\delta) \hat{\mathbf{d}}^{\prime}\right)$, where $\delta$ is thickness deviation from the expected thickness $\bar{t}$.

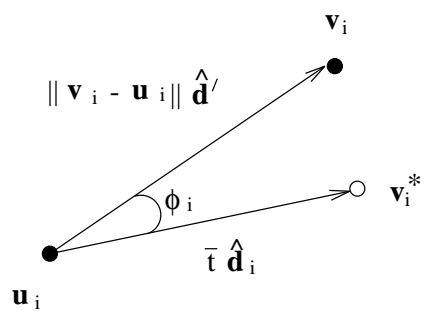

Figure 4: Notations used in the formulation of $e_{t h i c k} ; \mathbf{v}_{i}^{*}$ is the solution with minimum energy.

Property 1 constrains every snaxel $\mathbf{v}_{i} \in \mathcal{V}_{\text {out }}$ to be located at $\mathbf{v}_{i}^{*}=\mathbf{u}_{i}+\bar{t} \hat{\mathbf{d}}_{i}$, where $\mathcal{V}_{\text {in }}$ and $\mathcal{V}_{\text {out }}$ share similar shape and the separation between $\mathcal{V}_{\text {in }}$ and $\mathcal{V}_{\text {out }}$ is uniform over all snaxels. The remaining three properties penalize the snake for any discrepancy from the configuration constrained by Property 1 . Property 2 favors each outer snaxel $\mathbf{v}_{i}$ that is aligned to the direction specified by $\hat{\mathbf{d}}_{i}$. Property 3 ensures the separation between $\mathbf{v}_{i}$ and $\mathbf{u}_{i}$ to be as close to $\bar{t}$ as possible. Property 4 penalizes both positive and negative deviation from $\bar{t}$ by the same amount.

The energy functional that satisfies all the above properties is formulated as:

$$
\begin{aligned}
e_{\text {thick }}\left(\mathbf{v}_{i}\right) & =1-\left[1-\left(\frac{\left\|\mathbf{v}_{i}-\mathbf{u}_{i}\right\|-\bar{t}}{\bar{t}}\right)^{2}\right]\left|\cos \phi_{i}\right| \\
E_{\text {thick }}\left(\mathcal{V}_{\text {out }}\right) & =\sum_{i=1}^{n} e_{\text {thick }}\left(\mathbf{v}_{i}\right)
\end{aligned}
$$

The thickness deviation in Eq. (21) is normalized by the average thickness $\bar{t}$, thus this energy term is scale invariant. The energy profile specified by Eq. (21) represents a potential well with its minimum at $\mathbf{v}_{i}^{*}$. In addition, the equal thickness constrains the dynamic range of each snaxel $\mathbf{v}_{i}$ to that of $\mathbf{v}_{i}^{*}$. In summary, even in the absence of edge information on $\mathcal{V}_{\text {out }}$, an acceptable $\mathcal{V}_{\text {out }}$ boundary is attainable. 


\subsection{Energy Minimization Algorithm}

Different approaches have been proposed in the literatures to minimize the energy of snake. They all have a trade-off between the quality of solution and computational complexity. The method of variational calculus gives rise to a set of Euler equations which can be solved by iteration methods [14]. However, it suffers from numerical instability. Dynamic programming is stable and guarantees a global minimum, however, its computational cost is high for most practical applications [1]. The greedy algorithm, on the other hand, is very efficient, but it does not guarantee an optimal solution [24].

For our application, the greedy algorithm is adopted for its computational speed. Each updating in the greedy algorithm is chosen for local optimization which, however, does not gurantee a global optimal solution in the end. In our energy minimization process, a new updating rule is adopted to gurantee convergence of snakes.

Let $\mathcal{V}^{(k)}=\left\{\mathbf{v}_{i}^{(k)} \mid i=1,2, \cdots, n\right\}$ be the snake after the $k^{\text {th }}$ iteration. Its total energy is

$$
\begin{aligned}
E_{\text {snake }}^{(k)} & =E_{\text {snake }}\left(\mathcal{V}^{(k)}\right) \\
& =\sum_{i=1}^{n} e(i)
\end{aligned}
$$

where $e(i)$ is the energy of the $i^{t h}$ snaxel.

It can be verified from our energy Eqs. (17), (18), (20) and (21) that the energy e(i) depends on $\mathbf{v}_{i-1}$ and $\mathbf{v}_{i+1}$ in addition to $\mathbf{v}_{i}$. Once the $i^{\text {th }}$ snaxel is updated, $e(i-1), e(i)$ and $e(i+1)$ will be changed. Let $\hat{e}_{i}\left(\mathbf{v}_{i}^{(k)}\right)=e(i-1)+e(i)+e(i+1)$. Suppose each $\mathbf{v}_{i}^{(k)}$ generates a search window $W\left(\mathbf{v}_{i}^{(k)}\right)$ centered at $\mathbf{v}_{i}^{(k)}$ in which every position is a candidate point for updating. Our algorithm moves $\mathbf{v}_{i}^{(k)}$ to a new location $\mathbf{v}_{i}^{(k+1)}$ according to the following updating rule:

$$
\mathbf{v}_{i}^{(k+1)}=\arg \min _{\mathbf{v} \in W\left(\mathbf{v}_{i}^{(k)}\right)} \hat{e}_{i}(\mathbf{v})
$$

for $i=1,2, \cdots, n$. It can be shown that after each updating the new snake energy $E_{s n a k e}^{\prime}$ is less than the original energy $E_{\text {snake }}$, i.e.,

$$
\begin{aligned}
E_{\text {snake }}^{\prime} & =e(1)+\cdots+e(i-2)+\hat{e}_{i}\left(\mathbf{v}_{i}^{(k+1)}\right)+e(i+2)+\cdots+e(n) \\
& \leq e(1)+\cdots+e(i-2)+\hat{e}_{i}\left(\mathbf{v}_{i}^{(k)}\right)+e(i+2)+\cdots+e(n) \\
& =E_{\text {snake }}
\end{aligned}
$$

The inequality in Eq. (25) suggests that $\left\{E_{\text {snake }}^{(k)}\right\}$ is monotonically decreasing when the updating rule in Eq. (24) is applied. Due to the dependence of $e_{\text {cont }}$ and $e_{\text {thick }}$ on $\bar{d}$ and $\bar{t}$ respectively, $E_{\text {snake }}^{(k)}$ is also a function of the inter-snaxel spacing $\bar{d}^{(k)}$ and thickness $\bar{t}^{(k)}$. To be more precise, we write $E_{\text {snake }}^{(k)}$ as $E_{\text {snake }}\left(\mathcal{V}^{(k)}, \bar{d}^{(k)}, \bar{t}^{(k)}\right)$ where $\bar{d}^{(k)}$ and $\bar{t}^{(k)}$ are updated at every iteration. Although the updating Eq. (24) always results in a lower energy snake, updating of $\bar{d}^{(k)}$ and $\bar{t}^{(k)}$, in general, does not have the same guarantee. 
In order to guarantee convergence, $\bar{d}^{(k+1)}$ and $\bar{t}^{(k+1)}$ are updated such that $E_{\text {snake }}($ $\left.\mathcal{V}^{(k+1)}, \bar{d}^{(k+1)}, \bar{t}^{(k+1)}\right)$ is minimized over all $\bar{d}$ and $\bar{t}$, i.e.,

$$
E_{\text {snake }}\left(\mathcal{V}^{(k+1)}, \bar{d}^{(k+1)}, \bar{t}^{(k+1)}\right) \leq E_{\text {snake }}\left(\mathcal{V}^{(k+1)}, \bar{d}, \bar{t}\right)
$$

for any possible value denoted as $\bar{d}$ and $\bar{t}$, which ensures that the snake energy is nonincreasing. In this respect, $\bar{d}^{(k)}$ and $\bar{t}^{(k)}$ are interpreted as the optimal inter-snaxel spacing and thickness respectively, in the sense that the corresponding snake energy is minimized for the given snake configuration $\mathcal{V}^{(k)}$. The values of $\bar{d}^{(k+1)}$ and $\bar{t}^{(k+1)}$ are determined analytically by differentiation as follows. Let

$$
\left.\frac{\partial}{\partial d} E_{\text {snake }}\right|_{\bar{d}^{(k+1)}}=0
$$

then

$$
\bar{d}^{(k+1)}=\frac{\sum_{i=1}^{n}\left\|\mathbf{v}_{i}^{(k+1)}-\mathbf{v}_{i-1}^{(k+1)}\right\|^{2}}{\sum_{i=1}^{n}\left\|\mathbf{v}_{i}^{(k+1)}-\mathbf{v}_{i-1}^{(k+1)}\right\|}
$$

Let

$$
\left.\frac{\partial}{\partial t} E_{\text {snake }}\right|_{\bar{t}^{(k+1)}}=0
$$

then

$$
\bar{t}^{(k+1)}=\frac{\sum_{i=1}^{n}\left\{\left[t_{i}^{(k+1)}\right]^{2}\left|\cos \phi_{i}^{(k+1)}\right|\right\}}{\sum_{i=1}^{n}\left[t_{i}^{(k+1)}\left|\cos \phi_{i}^{(k+1)}\right|\right]}
$$

where $t_{i}^{(k+1)}=\left\|\mathbf{v}_{i}^{(k+1)}-\mathbf{u}_{i}^{(k+1)}\right\|$ and $\phi_{i}^{(k+1)}$ is the angle between $\mathbf{v}_{i}^{(k+1)}-\mathbf{u}_{i}^{(k+1)}$ and $\hat{\mathbf{d}}_{i}^{(k+1)}$.

Following the above procedure, we have established that the sequence $\left\{E_{\text {snake }}^{(k)}\right\}$ is monotonically decreasing, i.e.,

$$
E_{\text {snake }}^{(k+1)} \leq E_{\text {snake }}^{(k)}
$$

Moreover, $E_{\text {snake }}^{(k)}$ is bounded below by 0 , hence our algorithm converges in finite number of steps. If each search window $W\left(\mathbf{v}_{i}^{(k)}\right)$ consists of $m$ pixels, the proposed algorithm will terminate in $O(m n)$ time.

\section{CONFLICT RESOLUTION}

There are two types of error in the automated detection and extraction of axons; (i) misdetection and (ii) false detection. Misdetection is primarily due to the missing of interior points during the H-space search. False detection is caused by detection of potential interior points which are outside of the axon sheaths. Both under-counting and over-counting are 
not desirable. We have adopted a strategy to first over-count the number of potential axon interior points and to follow by eliminating those resulting snakes which are in conflict with other detected axons or lack physical evidence.

We denote the collection of candidate axons by a set $S=\left\{\mathcal{C}_{i} \mid i=1,2, \cdots, N\right\}$, where $\mathcal{C}_{i}=\left(\mathcal{V}_{i n}^{i}, \mathcal{V}_{\text {out }}^{i}\right)$. Obviously, the number of axons $N$ is controlled by the number of snakes that are released to the image, which in turn relies on the number of peaks detected in the H-space.

\subsection{Confidence Measure}

All correctly detected axons share some common physical characteristics which are distinct from falsely-detected axons. These physical characteristics are quantified by a set of features which are further transformed into a measure of physical evidence. The following lists the features that are used to differentiate true axons from false detection.

1. Average Intensity Gradient measures the average edge strength of all inner snaxel locations, i.e., $\sum_{i=1}^{n} \frac{\overline{\left|\nabla I\left(\mathbf{v}_{i}\right)\right|}}{n}$. Since inner boundaries are characterized by strong intensity gradients, this measure is typically close to 1 for true axons. In case of shot noise, the high intensity gradient due to the noise will be smoothed by averging. In general, the average intensity gradient decreases with $E_{\text {edge }}$ of the converged snake.

2. Compactness measures the regularity of a boundary by $\frac{4 \pi \text { Area }}{\text { (Perimeter }^{2}}$; it decreases with boundary irregularity. True axons are typically compact and smooth, therefore the compactness of their inner snakes is larger than that of false axons. It is to be noted that compactness is related to $E_{\text {cont }}$ and $E_{\text {curve }}$ where $E_{\text {cont }}$ and $E_{\text {curve }}$ decrease with increasing compactness measure.

3. Myelin Sheath Area is the ring enclosed by the inner and outer snakes, which consists of mostly pixels of low intensity. On the other hand, a falsely-detected sheath typically consists of background clutters and partial sheath of neighboring axons, thus the amount of low-intensity pixels enclosed by the snakes is comparatively small.

Let

$$
\begin{aligned}
& x_{1}=\text { average gradient intensity of an inner snake, } \\
& x_{2}=\text { compactness of an inner snake, and } \\
& x_{3}=\text { low intensity myelin sheath area }
\end{aligned}
$$

be the three components of a feature vector $\mathbf{x}$.

In order to have a simple scalar measure of physical evidence, $\mathbf{x}$ is transformed by $p=$ $\mathbf{w}^{t} \mathbf{x}$, where $\mathbf{w}$ is the projection vector. A training set of sample axons was used to determine $\mathbf{w}$, where $\mathbf{w}$ is chosen to maximize the separation between true axons and false axons by 
the Fisher's criterion [8]. The $p$ value is the measure of physical evidence based on each individual detected axons. It will be incorporated with a measure of conflict based on other neighboring axons, which is described in the following.

\subsection{Conflict Measure}

It has been observed that false alarms are typically detected between true axons as shown in Fig. 5(h). They are formed in part with background clutter and in part with sheaths from nearby axons. Since physical axons only touch each others and overlapping axons are physically impossible, any overlapping sheath detected between two axons must involve a false detection. A measure of conflict is deduced from overlapping axons to eliminate false detection.

Suppose that $\mathcal{C}_{i}$ overlaps with a set of candidate axons $\left\{\mathcal{C}_{j}\right\}$. Let $A_{i}$ be the area of the sheath of $\mathcal{C}_{i}$, and $A_{i j}$ be the area of overlapping sheath between $\mathcal{C}_{i}$ and $\mathcal{C}_{j}$. The ratio $\frac{A_{i j}}{A_{i}}$, bounded between 0 and 1 , measures the amount of conflict between $\mathcal{C}_{i}$ and $\mathcal{C}_{j}$. The conflict measure between $\mathcal{C}_{i}$ and $\left\{\mathcal{C}_{j}\right\}$ is defined as

$$
c_{i}=\sum_{j} p_{j} Q\left(\frac{A_{i j}}{A_{i}}\right)
$$

The function $Q\left(\frac{A_{i j}}{A_{i}}\right)$ represents the amount of inconsistency arising from $\mathcal{C}_{i}$ and $\mathcal{C}_{j}$, which will be defined in the Appendix.

The value of $Q\left(\frac{A_{i j}}{A_{i}}\right)$ is further weighted by $p_{j}$, the confidence measure of physical evidence of $\mathcal{C}_{j}$, to emphasize the conflict if the overlapping axon is a potential true axon indicated by a large $p_{j}$, or to de-emphasize $c_{i}$ if $\mathcal{C}_{j}$ is a potential false detection with a small $p_{j}$.

The physical evidence $p_{i}$ strengthens our confidence about $\mathcal{C}_{i}$ while the conflict measure $c_{i}$ diminishes our belief about $\mathcal{C}_{i}$. These two sources of information are finally combined into a single confidence measure $q_{i}$ given by

$$
\begin{aligned}
q_{i} & =p_{i}-c_{i} \\
& =p_{i}-\sum_{j} p_{j} Q\left(\frac{A_{i j}}{A_{i}}\right)
\end{aligned}
$$

which incorporates both the local information of $\mathcal{C}_{i}$ and the interaction between $\mathcal{C}_{i}$ and its neighboring axons $\left\{\mathcal{C}_{j}\right\}$.

\subsection{False Alarm Elimination}

The total confidence on the set of axons $S$ is defined as

$$
J_{S}=\sum_{i=1}^{N} q_{i}
$$


It can be shown that $J_{S}$ increases significantly as false alarms are being eliminated from $S$. In contrary, the removal of a true axon in general reduces the value of $J_{S}$, or at worst causes a small numerical fluctuation of $J_{S}$, thus $J_{S}$ is a reliable indication of false alarm removal. A decision variable $s_{i}$ is further defined:

$$
s_{i}= \begin{cases}1 & \text { if } \mathcal{C}_{i} \text { is taken as a true axon } \\ 0 & \text { if } \mathcal{C}_{i} \text { is taken as a false axon }\end{cases}
$$

The collection of all true axons, denoted as a set $\hat{S} \subseteq S$, has the following confidence for $\operatorname{each} \mathcal{C}_{i}$ in $\hat{S}$

$$
q_{i}=p_{i}-\sum_{j} s_{j} p_{j} Q\left(\frac{A_{i j}}{A_{i}}\right)
$$

The total confidence associated with the set $\hat{S}$ becomes

$$
\begin{aligned}
J_{\hat{S}} & =\sum_{i=1}^{N} s_{i} q_{i} \\
& =\sum_{i=1}^{N} s_{i}\left[p_{i}-\sum_{j} s_{j} p_{j} Q\left(\frac{A_{i j}}{A_{i}}\right)\right] \\
& =\sum_{i=1}^{N} s_{i} p_{i}-\sum_{i=1}^{N} \sum_{j} s_{i} s_{j} p_{j} Q\left(\frac{A_{i j}}{A_{i}}\right)
\end{aligned}
$$

Eq. (37) can be viewed as a criterion function with variable $s_{i}$ which is the label for the axons in $\hat{S}$. The objective becomes switching $s_{i}$, which is equivalent to eliminating $\mathcal{C}_{i}$, such that $J_{\hat{S}}$ becomes most confident. In other words, what is the assignment of $s_{i}$ 's such that the total confidence is maximized? Mathematically, we formulate our objective as

$$
\max J\left(s_{1}, s_{2}, \cdots, s_{N}\right)=\sum_{i=1}^{N} s_{i} p_{i}-\sum_{i=1}^{N} \sum_{j} s_{i} s_{j} p_{j} Q\left(\frac{A_{i j}}{A_{i}}\right)
$$

Furthermore, Eq. (38) can be written in a more familiar form by introducing the variable $s_{i j}$ and the respective equality constraints:

$$
\max J=\sum_{i=1}^{N} s_{i} p_{i}-\sum_{i=1}^{N} \sum_{j} s_{i j} p_{j} Q\left(\frac{A_{i j}}{A_{i}}\right)
$$

subject to

$$
\begin{aligned}
s_{i j} & =s_{i} s_{j} \quad \text { for } i=1, \cdots, N, \\
s_{i} & =0 \text { or } 1 \quad \text { for } i=1, \cdots, N
\end{aligned}
$$

The above formulation is in the form of a 0-1 integer programming problem and hence can be solved by the branch-and-bound algorithm [9]. One drawback of the branch-and-bound 
algorithm is that its complexity grows rapidly with the number of inputs. For an image with a few hundreds of axons, its processing time will become impractical. However, the amount of computation is drastically reduced when the set $S$ is partitioned into several independent subsets, each of which has no relation with others. In other words, the labelling made in one subset has no bearing to any other subsets. This applies directly to the analysis of axon images in which overlapping axons are clustered together, each forming an independent subset.

Let us model each $\mathcal{C}_{i}$ as a vertex $i$ of a graph $G$. If $\mathcal{C}_{i}$ overlaps with $\mathcal{C}_{j}$, an edge is drawn to connect vertex $i$ and vertex $j$. The graph $G$ represents the spatial relationship of axons in $S$. Suppose that $\mathcal{C}_{i}$ and $\mathcal{C}_{j}$ are axons of different independent subsets, there should be no path in $G$ connecting vertex $i$ and vertex $j$. Therefore, each independent subset, in fact, corresponds to a connected component in $G$, i.e., an overlapping cluster of axons. The entire $S$ can be partitioned by an graph searching algorithm, such as the depth-first search. After partitioning, the labelling problem in Eq. (39) is solved for each independent subset separately, thus significantly reduces the input size of each instance of the branch-and-bound algorithm.

\section{EXPERIMENTS}

\subsection{Physical Measurements}

To count the number of axons in a complete nerve, the electronmicrograph image must be segmented into overlapping frames, each to be processed by the proposed analysis algorithm. Axons at or near the border of each frame will lie partly inside and partly outside. In order to avoid over-counting, only axons that lie entirely within the image frame are counted. That is, every axon in $\hat{S}$ is first extracted and those which extend beyond the image frame are removed. A correct count of the entire nerve can then be achieved by a frame overlapping scheme such that all axons will be accounted for.

The axon size is computed using the inner snake of each detected axon. The inner snake is approximated by a polygon whose vertices are snaxels. The area of the polygon is directly calculated as:

$$
\text { area }=\frac{1}{2}\left|\sum_{i=1}^{n}\left(x_{i} y_{i+1}-x_{i+1} y_{i}\right)\right|
$$

where $\left(x_{i}, y_{i}\right)$ denotes the coordinates of a vertex and by convention, $\left(x_{n+1}, y_{n+1}\right)=\left(x_{1}, y_{1}\right)$ [21].

Axon dimensions are estimated from the major and minor axes of an ellipse appropriately fitted to the region enclosed by the inner snake. The best-fit ellipse is chosen as the one whose second moment equals to that of the axon and the lengths of its axes indicate the size. The 
lengths of the semi-major and semi-minor axes, $a$ and $b$ respectively, of the best-fit ellipse are computed by the following [12]

$$
\begin{aligned}
\theta & =\frac{1}{2} \tan ^{-1}\left(\frac{2 m_{1,1}}{m_{2,0}-m_{0,2}}\right) \\
I_{\text {min }} & =\sum_{(x, y) \in R}[(y-\bar{y}) \cos \theta-(x-\bar{x}) \sin \theta]^{2} \\
I_{\text {max }} & =\sum_{(x, y) \in R}[(y-\bar{y}) \sin \theta+(x-\bar{x}) \cos \theta]^{2} \\
a & =\left(\frac{4}{\pi}\right)^{\frac{1}{4}}\left[\frac{\left(I_{\text {max }}\right)^{3}}{I_{\min }}\right]^{\frac{1}{8}} \\
b & =\left(\frac{4}{\pi}\right)^{\frac{1}{4}}\left[\frac{\left(I_{\min }\right)^{3}}{I_{\max }}\right]^{\frac{1}{8}}
\end{aligned}
$$

where $R$ is the region bounded by an inner snake, $m_{i, j}$ denotes the central moment of order $(i, j)$ and $(\bar{x}, \bar{y})$ is the centroid of $R$.

Finally, the thickness of myelin sheath is estimated by the separation between the inner and outer snakes of detected axons. For each inner snaxel, the perpendicular distance between that and the outer boundary is measured. Let this length be $t_{i}$, then the average thickness is

$$
\text { average thickness }=\frac{\sum_{i=1}^{n} t_{i}}{n}
$$

\subsection{Specifications of Inner Snakes and Outer Snakes}

The total energy of inner snake $\mathcal{V}_{i n}$ is given by

$$
E_{\text {snake }}\left(\mathcal{V}_{\text {in }}\right)=\lambda_{\text {cont }} E_{\text {cont }}\left(\mathcal{V}_{\text {in }}\right)+\lambda_{\text {curve }} E_{\text {curve }}\left(\mathcal{V}_{\text {in }}\right)+\lambda_{\text {edge }} E_{\text {edge }}\left(\mathcal{V}_{\text {in }}\right)
$$

Since most inner boundaries are continuous and exhibit strong intensity gradients, we assign

a large value to $\lambda_{e d g e}$, thus giving image intensity data a high degree of influence. The regularization parameters were determined experimentally for the best average results using a training set of sample images; they are: $\lambda_{\text {cont }}=0.24, \lambda_{\text {curve }}=0.16$ and $\lambda_{\text {edge }}=0.60$.

On the contrary, intensity gradient is less reliable regarding outer boundary detection due to the fact that many axons are touching one another with unresolved boundaries. Moreover, there are background clutters in between axons which will mislead the convergence of the snake. If the regularization parameters are not chosen properly, the outer snake of one axon may converge to include parts of an adjacent fiber. With this consideration in mind, the total energy of outer snake $\mathcal{V}_{\text {out }}$ is specified to be

$$
\begin{aligned}
E_{\text {snake }}\left(\mathcal{V}_{\text {out }}\right)= & \lambda_{\text {cont }} E_{\text {cont }}\left(\mathcal{V}_{\text {out }}\right)+\lambda_{\text {curve }} E_{\text {curve }}\left(\mathcal{V}_{\text {out }}\right)+ \\
& \lambda_{\text {thick }} E_{\text {thick }}\left(\mathcal{V}_{\text {out }}\right)+\lambda_{\text {edge }} E_{\text {edge }}\left(\mathcal{V}_{\text {out }}\right)
\end{aligned}
$$


and the regularization parameters were determined experimentally using sample images to be $\lambda_{\text {cont }}=0.12, \lambda_{\text {curve }}=0.20, \lambda_{\text {thick }}=0.48$ and $\lambda_{\text {edge }}=0.2$. Figure $2(\mathrm{~g})$ shows the converged snake $\mathcal{V}_{\text {out }}$. It is to be noted that $\mathcal{V}_{\text {out }}$ fails to detect a part of the right boundary due to the change in sheath thickness. However, the regularization parameter $\lambda_{\text {thick }}=0.48$ could be reduced to a smaller value and $\lambda_{e d g e}=0.2$ could be increased so that the change in sheath thickness could be captured by $\mathcal{V}_{\text {out }}$.

\section{$8.3 \quad$ Results}

The proposed algorithm has been tested using 10 frames of a nerve image. They contain a total of 196 axons. The sequence given in Fig. 5 shows the intermediate results of the algorithm. The computation time for processing Fig. 5, which contains 48 true axons, is about 7 minutes on a Sun Sparc 10 workstation. 


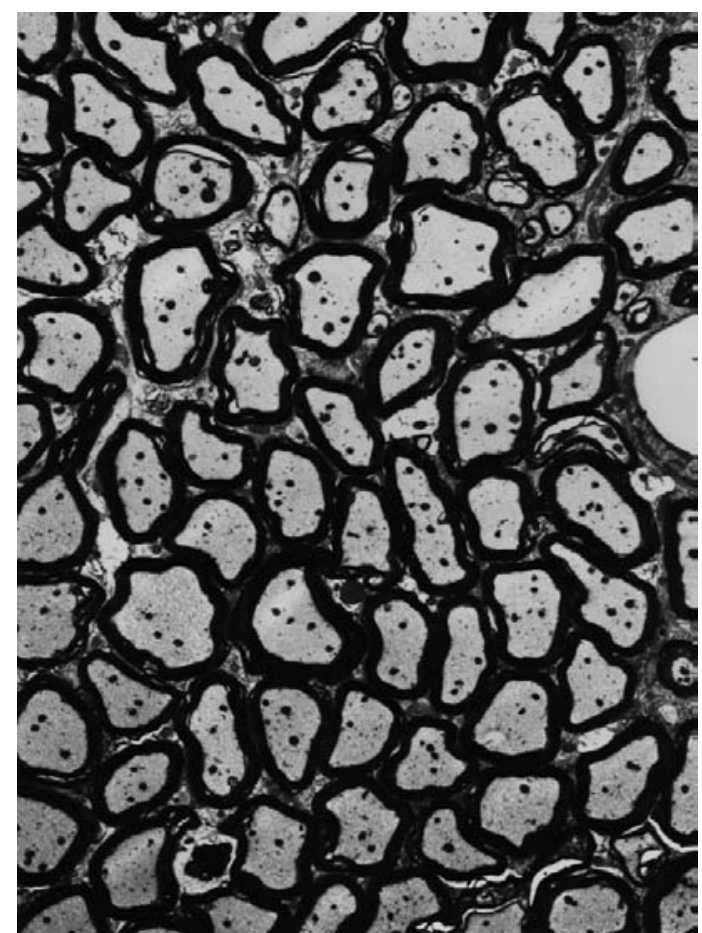

5(a) Input image (x9000).

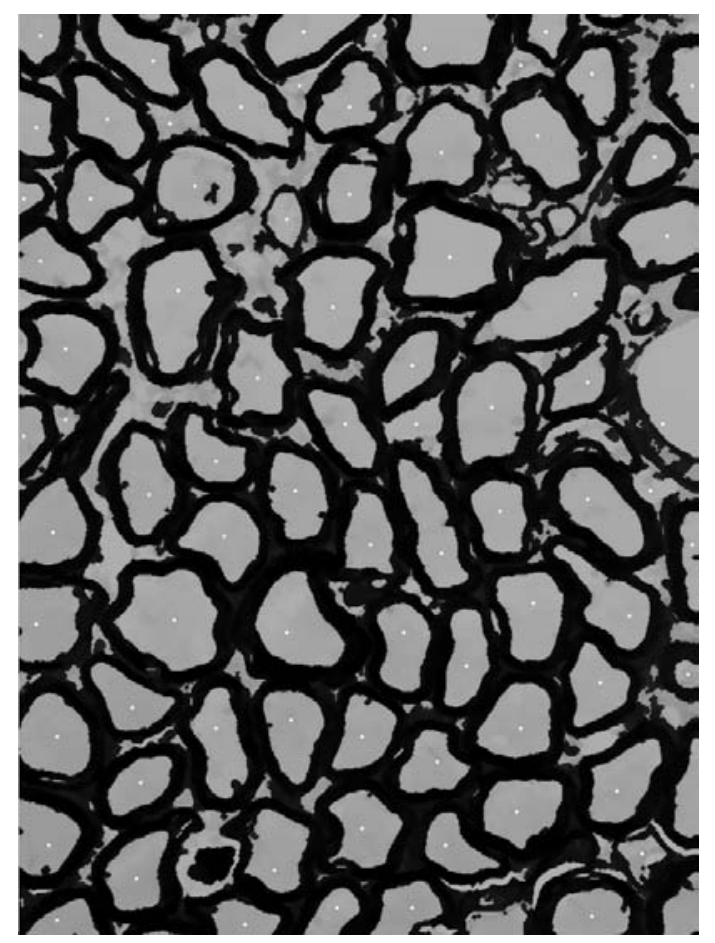

5(c) Detected interior points.

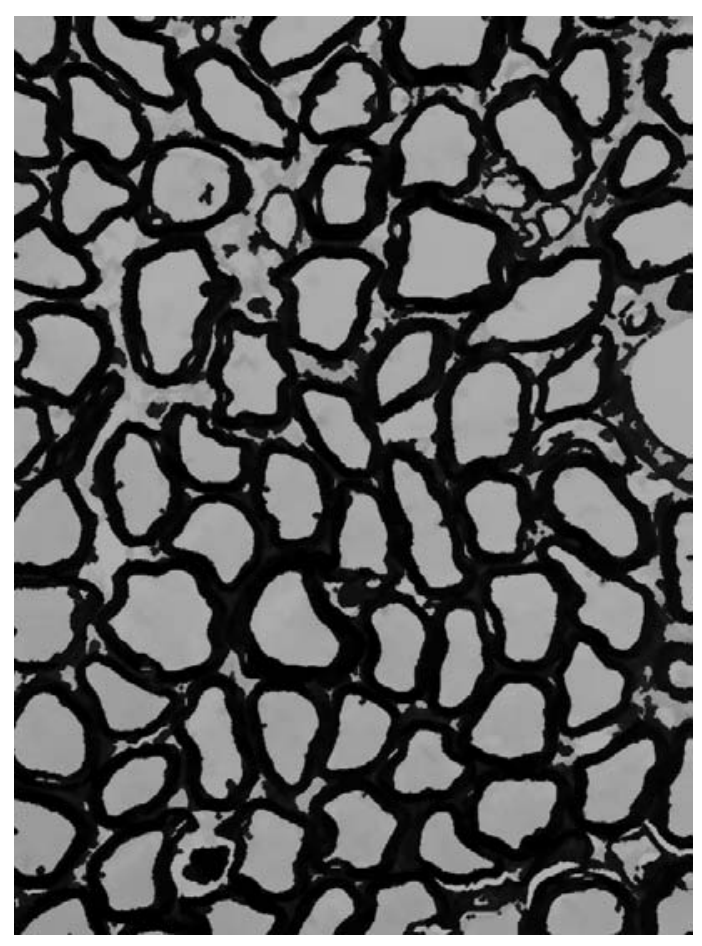

5(b) Preprocessed image.

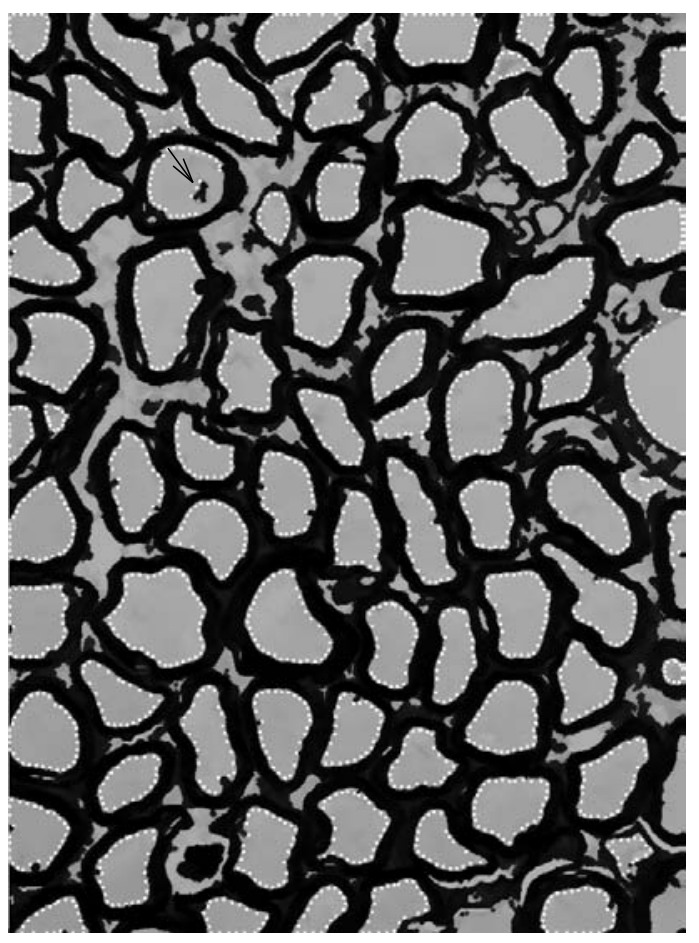

5(d) Initial inner snakes. 


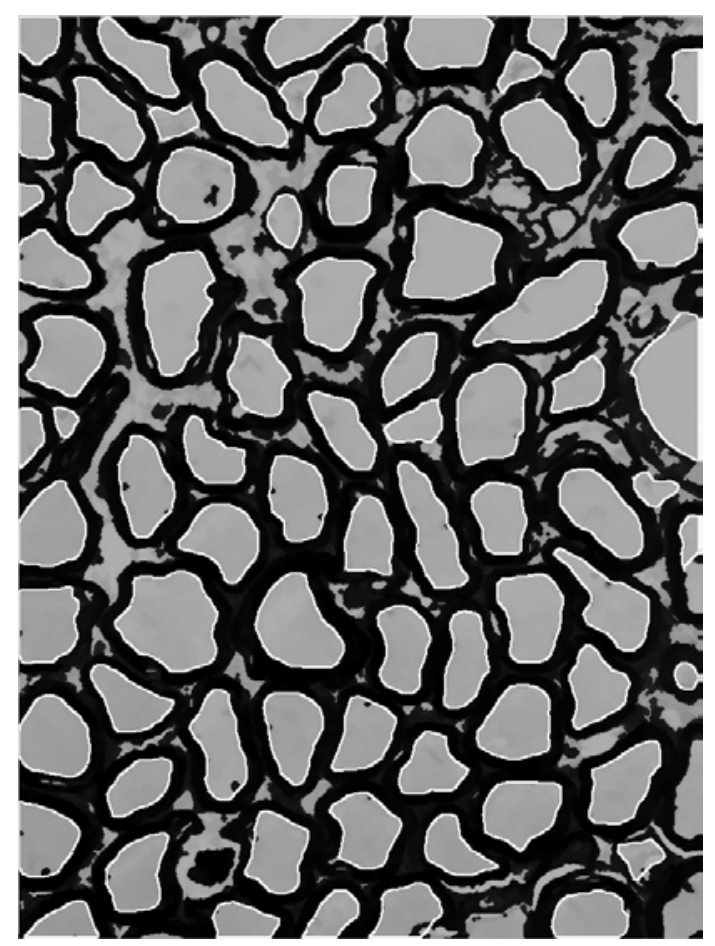

5(e) Converged inner snakes.

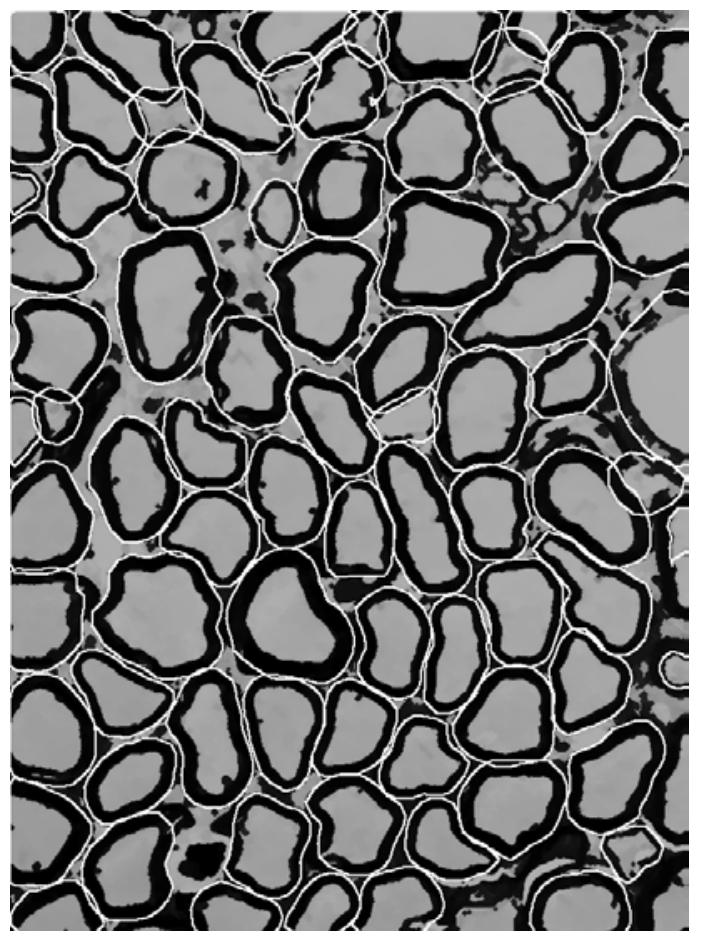

5(g) Converged outer snakes.

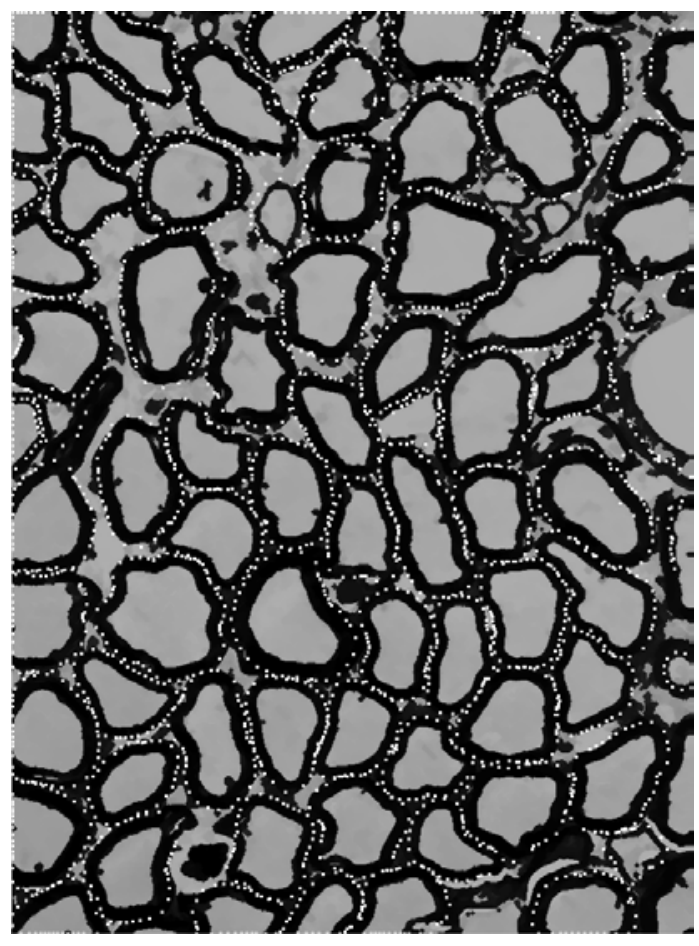

5(f) Initial outer snakes.

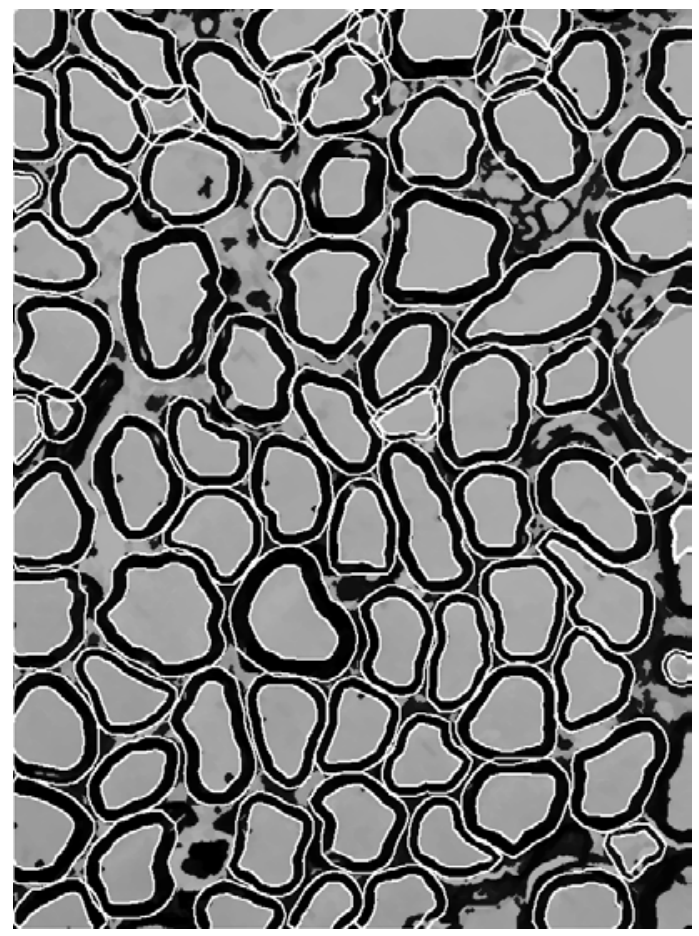

5(h) All detected axons. 


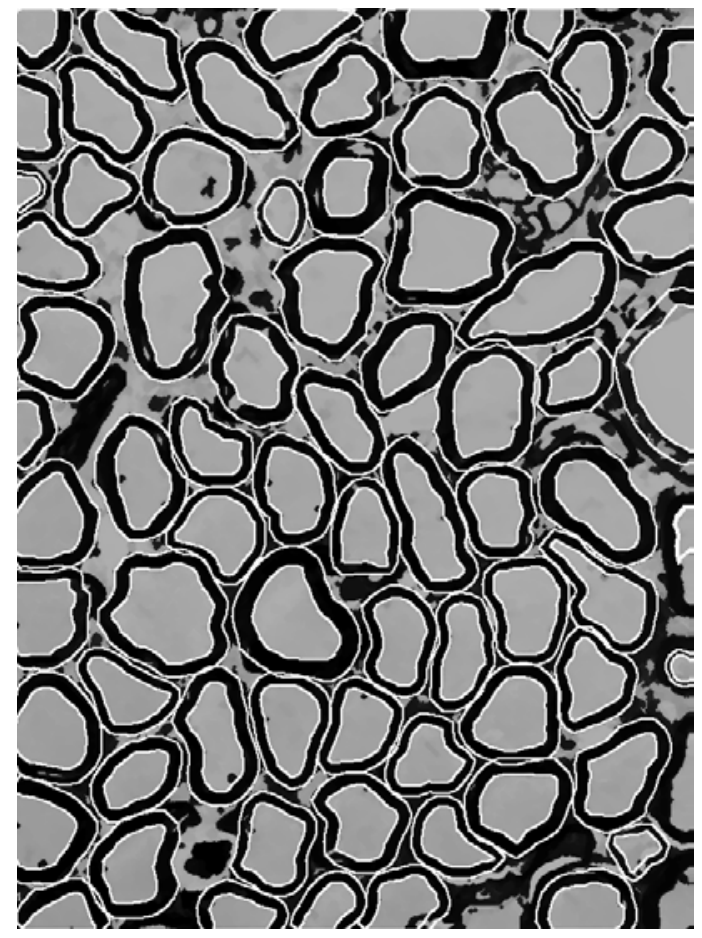

5(i) Remaining axons after conflict resolution.

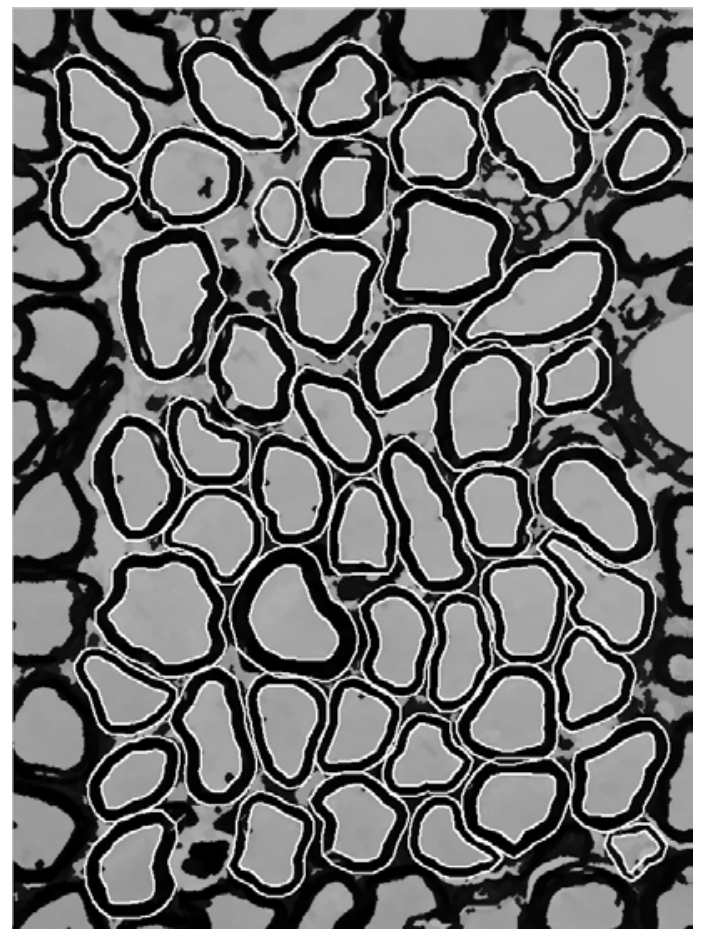

5(j) Partial axons removed.

Figure 5: Detection results to illustrate the processing sequence.

Fig. 5(b) shows the removal of axoplasmic organelles like mitochondria by the multidirectional filter in the preprocessing stage. It can be seen from Fig. 5(c) that the algorithm detects potential interior points from both true axons and false axons. Figs. 5(d) and 5(e) show that almost all inner snakes describe the axon boundaries accurately. For axons whose initial inner snakes are misled by axoplasmic organelles (see an example indicated by an arrow), their final snakes are still able to converge to locate the true physical boundaries based on the energy minimization process. Fig. 5(f) shows the outer snake initialization process; initial outer snakes resemble the shape of their respective inner boundaries. By the equal thickness constraint, the outer snakes converge accurately even in cases of incomplete boundary information. Fig. 5(h) shows a number of false detections that occur frequently between axons. Fig. 5(i) illustrates the results of the conflict resolution process - most false detections are eliminated while true axons are preserved. Finally, Fig. 5(j) shows the final set of detected axons. ${ }^{6}$

Detection results of some other images are shown in Fig. 6 and their aggregate size distributions are shown in Fig. 7.

\footnotetext{
${ }^{6}$ There are occasional overlapping boundaries in the final detected result which are detection artifacts. See the upper right-hand corner in $5(\mathrm{j})$. An alternate implementation is to re-define the boundary as midway between the two overlapped portions. This has not been done in the current implementation.
} 


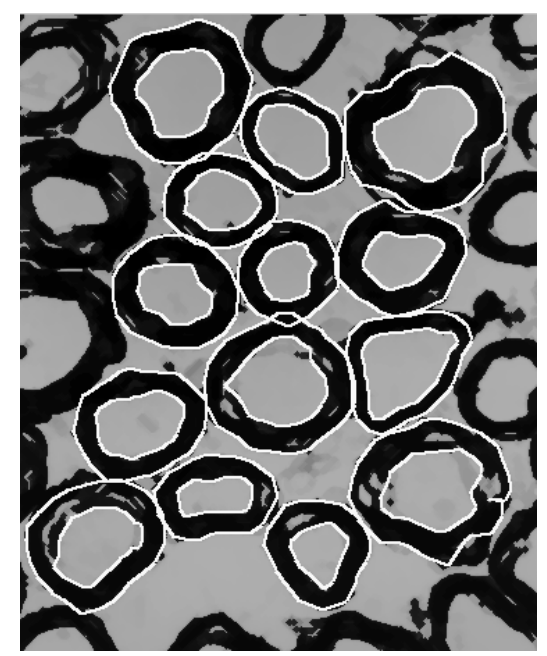

6(a) Detected axons with holes in myelin sheaths.

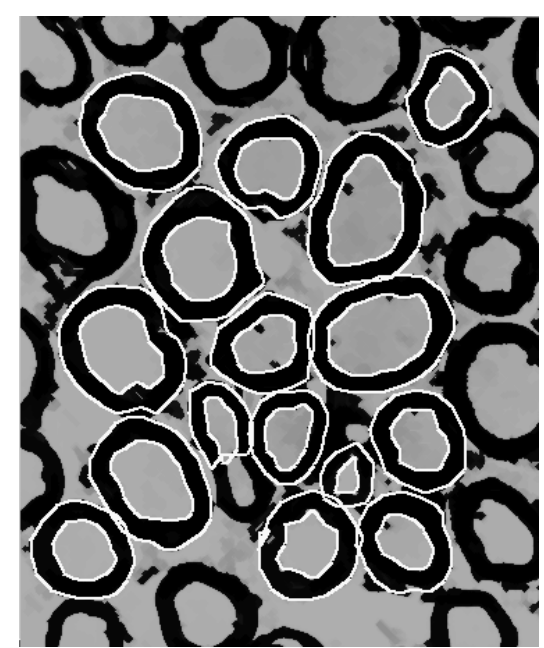

6(b) Error in detection due to elongated axon shape. 


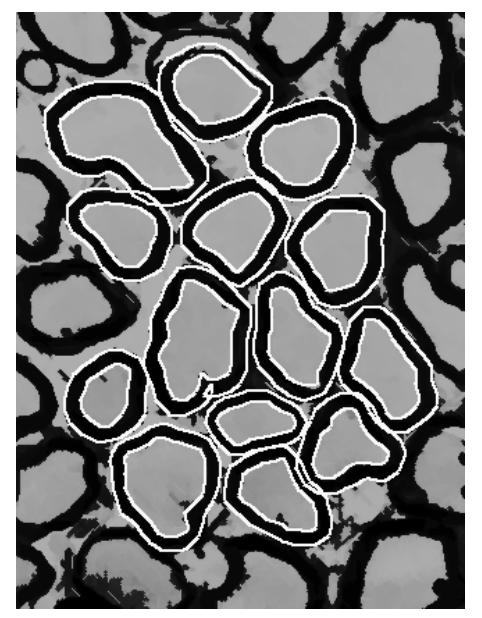

6(c) Detected axons with adjoined boundaries.

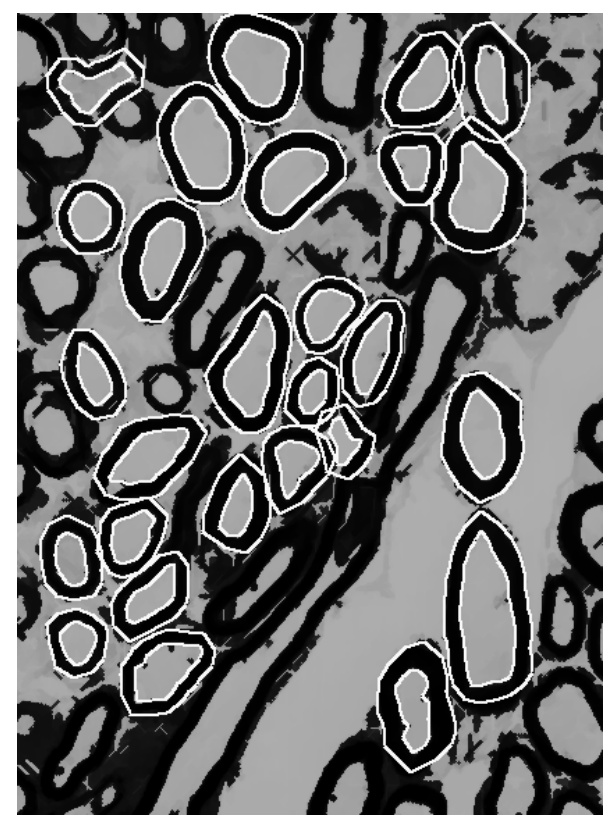

6(d) Misdetection of elongated shapes and small axons.

Figure 6: Additional Detection results.

Apart from cases like the image in Fig. 6(d), the proposed nerve-cell image analyzer is able to detect and measure axons accurately. The principal limitation in performance is shown in the last image (Fig. 6(d)). For axons that are much smaller than the typical size, the peaks cast by them in the H-space interior point localization are strongly interfered by nearby structures. These peaks are easily buried in the noise of the $\mathrm{H}$-space, and hence the algorithm fails to detect their presence. Furthermore, while the algorithm is unable to locate these small axons, their boundaries act as structured noise which interferes with the detection of other axons and leads to false alarms. The false alarms that are close to the undetected small axons are extremely hard to be eliminated since there is no conflict associated with 


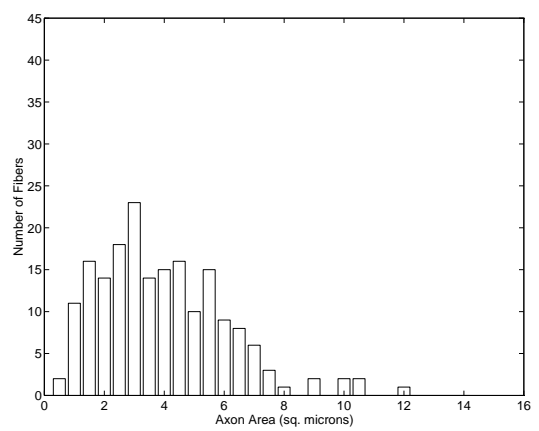

(a)

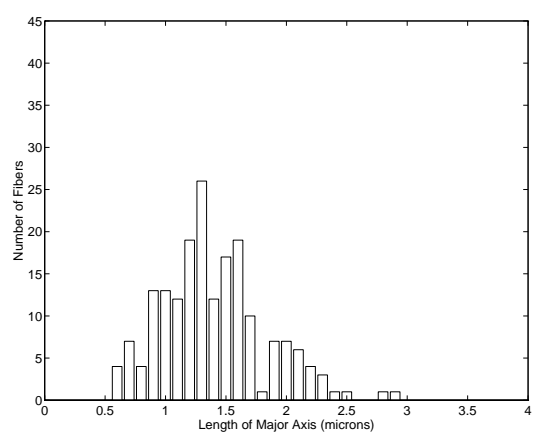

(c)

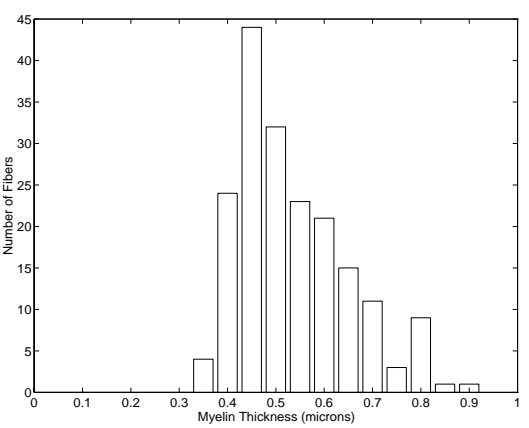

(b)

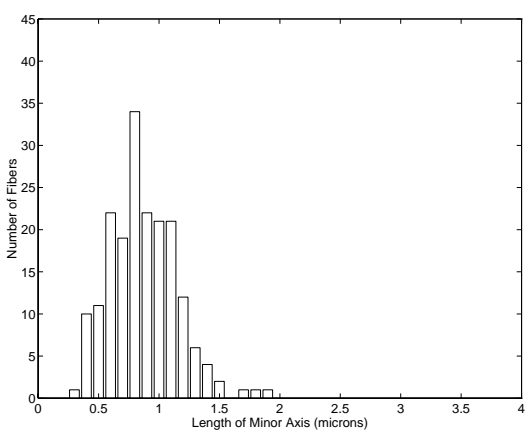

(d)

Figure 7: The aggregate size distributions 
such cases. Fig. 7 shows the size distributions of all the axons detected. The results are comparable to those done manually reported in [3].

Table 1 tabulates the detection result and Table 2 summarizes the performance. The detection rate is defined as the ratio of the number of detected true axons to the total number of axons, excluding the count due to false detection. The false alarm rate is the ratio of the number of false detections to the number of axons detected. The overall detection rate and false alarm rate using this set of images are found to be $93 \%$ and $3 \%$ respectively.

\begin{tabular}{|l||c|c|c|c|}
\hline Image ID & \# of fibers & \# of entities detected & \# of true fibers & \# of false alarms \\
\hline \hline 1 (Fig. 6(a)) & 14 & 14 & 14 & 0 \\
\hline 2 (Fig. 6(b)) & 16 & 16 & 16 & 0 \\
\hline 3 (Fig. 5(j)) & 48 & 49 & 48 & 1 \\
\hline 4 & 13 & 13 & 13 & 0 \\
\hline 5 & 12 & 12 & 12 & 0 \\
\hline 6 & 16 & 16 & 16 & 0 \\
\hline 7 (Fig. 6(c)) & 14 & 14 & 14 & 0 \\
\hline 8 & 9 & 9 & 9 & 0 \\
\hline 9 & 22 & 18 & 16 & 2 \\
\hline 10 (Fig. 6(d)) & 32 & 27 & 25 & 5 \\
\hline \hline Total & 196 & 188 & 183 & 2 \\
\hline
\end{tabular}

Table 1: Detection Results

\section{CONCLUSION}

A human brain consists of billions of nerve cells interconnected by their projection fibers called axons. It is believed that the number of axons, their size and shape are essential in understanding the normal or abnormal, the developing or aging, as well as the injured or diseased brains. The deterioration of the myelin sheath wrapping will also cause various diseases, for example, multiple sclerosis. In addition, the axon diameter is known to affect the conduction property of the axons which in turn affects the brain functions. Therefore, a quantitative knowledge of the number of axons, as well as their size and shape, is essential in quantitative analysis of the brain.

A system for automatic counting and sizing of axons is presented in this paper. The core of our system is based on a boundary detection technique called the active contour model (snakes). The advantage of this method over traditional approaches (e.g., edge tracing and Hough transform) is attributed to the fact that it integrates both data-driven and model- 


\begin{tabular}{|l||c|c|}
\hline Image ID & Detection rate (\%) & False alarm rate (\%) \\
\hline \hline 1 (Fig. 6(a)) & 100 & 0 \\
\hline 2 (Fig. 6(b)) & 100 & 0 \\
\hline 3 (Fig. 5(j)) & 100 & 2 \\
\hline 4 & 100 & 0 \\
\hline 5 & 100 & 0 \\
\hline 6 & 100 & 0 \\
\hline 7 (Fig. 6(c)) & 100 & 0 \\
\hline 8 & 100 & 0 \\
\hline 9 & 73 & 13 \\
\hline 10 (Fig. 6(d)) & 78 & 8 \\
\hline \hline Overall & 93 & 3 \\
\hline
\end{tabular}

Table 2: System Performance

driven approaches in a concise manner. We have addressed three issues specific for this application. They include: (1) Snake initialization, (2) energy functional formulation, and (3) elimination of false detection.

A Hough transform approach, based on an elliptical approximation to the inner boundaries, was used for the detection of all potential axons. Local evidence is accumulated in an accumulator array called H-space for the most likely candidates, thereby generating a set of axon interior points, each of which is a potential detection. This method is robust to image noise and irrelevant image features. Initial inner snakes are constructed by growing outward from the interior points. Initial outer snakes are created by uniformly expanding the converged inner snake $t^{*}$ distance outward. The optimal distance $t^{*}$ for each axon is estimated by using the edge strength distribution estimated from the corresponding inner boundary.

An energy functional was formulated to match the outer snakes to the appearance of the inner snakes by a uniform thickness constraint whereby missing boundaries are interpolated accurately. Overlapping among neighboring axons is physically impossible, thus such detection is considered to be error and is used to eliminate false alarms. A confidence measure based on overlapping axons and physical axon properties is optimized in a conflict resolution algorithm by 0-1 integer programming. This enables the accurate detection of axons with minimal false detection.

Our proposed algorithm has been tested against a set of real images. The detection rate and false alarm rate are found to be $93 \%$ and $3 \%$ respectively. The remaining source of error 


\section{Appendix: The $Q$ function}

The $Q$ function in Eq. (32) maps the amount of axon overlap into a measure of inconsistency. This mapping is not absolutely required for the implementation of conflict resolution, however, an appropriate $Q$ function enhances the confidence measure.

First, true axons do not overlap physically, so the $Q$ function should be insensitive to little or no overlap. Second, a false axon usually overlaps with more than one neighboring axons, $Q$ should therefore map into a high value when a large number of adjacent axons are involved in the overlapping.

The following $Q$ function, which possesses the above two properties, is defined to be

$$
Q(x)=\frac{1}{K}\left\{\tanh \left[\beta\left(x-x_{0}\right)\right]+K_{0}\right\}
$$

where

$$
\begin{aligned}
K_{0} & =\tanh \left(\beta x_{0}\right) \\
K & =\tanh \left[\beta\left(1-x_{0}\right)\right]+\tanh \left(\beta x_{0}\right)
\end{aligned}
$$

It is a hyperbolic tangent function shifted vertically by $K_{0}$ and scaled by $K$, where $Q(0)=$ $0, Q(1)=1$ and $Q(x)$ is monotonically increasing. The parameter $x_{0}$ denotes the critical point $-Q(x)$ suppresses the inconsistency when $x<x_{0}$ and amplifies it when $x>x_{0}$. The parameter $\beta$ controls the steepness of the curve near $x_{0}$. By adjusting the parameters $x_{0}$ and $\beta$, we can control the operating range to enhance the conflict resolution algorithm. Note that

$$
Q\left(x^{\prime}+x^{\prime \prime}\right)<Q\left(x^{\prime}\right)+Q\left(x^{\prime \prime}\right)
$$

when $x^{\prime}>x_{0}$ and $x^{\prime \prime}>x_{0}$. This implies that the conflict measure increases with the number of overlapping axons involved. Figure 8 shows the $Q$ function used in the algorithm.

\section{References}

[1] A. A. Amini, T. E. Weymouth, and R. C. Jain, "Using dynamic programming for solving variational problems in vision," IEEE Trans. Pattern Analysis and Machine Intelligence, vol. 12 , no. 9, pp. 855-867, 1990.

[2] D. H. Ballard, "Generalizing the Hough transform to detect arbitrary shapes," Pattern Recognition, vol. 13, pp. 111-122, 1981. 


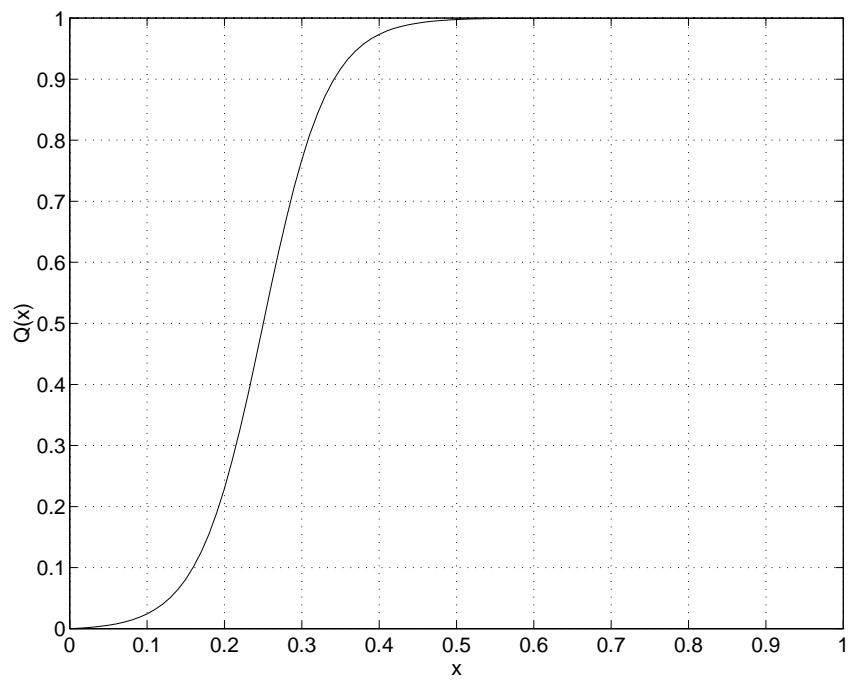

Figure 8: The $Q$ function used in the conflict resolution algorithm.

[3] A. Barbary, "Auditory nerve of the normal and jaundiced rat. I. Spontaneous discharge rate and cochlear nerve histology," Hearing Research, pp. 75-90, 1991.

[4] M. Berger and R. Mohr, "Towards autonomy in active contour models," 11th IAPR International Conference on Pattern Recognition, pp. 847-851, 1990.

[5] L. D. Cohen, "On active contour models and balloons," Computer Vision, Graphics and Image Processing-Image Understanding, vol. 53, no. 2, pp. 211-218, 1991.

[6] I. Cseke, "A fast segmentation scheme for white blood cell images," 11th IA PR International Conference on Pattern Recognition, vol. III, pp. 530-533, 1992.

[7] C. Domengeaux and J. C. F. Dewey, "Cell shape analysis by gradient methods," The 6th Scandinavian Conference on Image Analysis, pp. 349-354, 1989.

[8] R. O. Duda and P. E. Hart, "Use of the Hough transform to detect lines and curves in pictures," Communs. Ass. comput. Mach, vol. 15, pp. 11-15, 1972.

[9] J. G. Ecker and M. Kupferschmid, Introduction to operations research. New York: Wiley, 1988.

[10] A. Elmoataz, M. Revenu, and C. Porquet, "Segmentation and classification of various types of cells in cytological images," International Conference on Image Processing and its Applications, pp. 385-388, 1992.

[11] C. Garbay, "Image structure representation and processing: A discussion of some segmentation methods in cytology," IEEE Trans. Pattern Analysis and Machine Intelligence, vol. 8, no. 2, pp. 140-146, 1986.

[12] A. K. Jain, Fundamentals of Digital Image Processing. Prentice-Hall, 1989. 
[13] A. K. Jain, S. P. Smith, and E. Backer, "Segmentation of muscle cell pictures: A preliminary study," IEEE Trans. Pattern Analysis and Machine Intelligence, vol. 2, no. 3, pp. $232-242,1980$.

[14] M. Kass, A. Witkin, and D. Terzopoulos, "Snakes: Active contour models," Proceedings of First International Conference on Computer Vision, pp. 259-269, 1987.

[15] K. F. Lai and R. T. Chin, "On regularization, formulation and initialization of the active contour models (snakes)," Asian Conference on Computer Vision, pp. 542-545, 1993.

[16] S. Menet, P. Saint-Marc, and G. Medioni, "Active contour models: Overview, implementation and applications," IEEE Int. Conf. on Systems, Man and Cybernetics, pp. 924-929, 1990 .

[17] J. K. Mui, K. S. Fu, and J. W. Bacus, "Automated classification of blood cell neutrolphils," J. Histochem. Cytochem, vol. 25, pp. 633-640, 1977.

[18] P. Mussio, M. Pietrogrande, P. Bottoni, M. Dell'Oca, E. Arosio, E. Sartirana, M. R. Finanzon, and N. Dioguardi, "Automatic cell count in digital images of liver tissue sections," Proceedings of the Fourth Annual IEEE Symposium, pp. 153-160, 1991.

[19] L. O’Gorman and A. C. Sanderson, "The wedge filter technique for convex boundary estimation," IEEE Trans. Pattern Analysis and Machine Intelligence, vol. 7, no. 3, pp. 326-332, 1985 .

[20] J. M. S. Prewitt and M. L. Mendelsohn, "The analysis of cell images," Ann. N. Y. Acad. Sci., vol. 128, pp. 1035-1053, 1966.

[21] J. C. Russ, Computer-Assisted Microscopy - The measurement and Analysis of Images. New York: Plenum Press, 1990.

[22] F. Safa and G. Flouzat, "Speckle removal on radar imagery based on mathematical morphology," Signal Processing, vol. 16, pp. 319-333, 1989.

[23] D. Terzopoulos, "Regularization of inverse visual problems involving discontinuitites," IEEE Trans. Pattern Analysis and Machine Intelligence, vol. 8, no. 4, pp. 413-424, 1986.

[24] D. J. Williams and M. Shah, "A fast algorithm for active contours and curvature estimation," Computer Vision, Graphics, Image Processing, vol. 55, pp. 14-26, 1992.

[25] H. K. Yuen, J. Illingworth, and J. Kittler, "Detecting partially occluded ellipses using the hough transform," Image and vision computing, vol. 7, no. 1, pp. 31-37, 1989.

[26] S. Zucker, C. David, A. Dobbins, and L. Iversion, "The organization of curve detection: Coarse tangent fields and fine spline coverings," Proceedings of Second International Conference on Computer Vision, pp. 568-577, 1988. 